\title{
MiR-16 regulates crosstalk in NF-kB tolerogenic inflammatory signaling between myeloma cells and bone marrow macrophages
}

\author{
Jihane Khalife, ${ }^{1,2}$ Jayeeta Chose, ${ }^{3}$ Marianna Martella, ${ }^{1,2}$ Domenico Viola, ${ }^{1,2}$ Alberto Rocci, ${ }^{4}$ \\ Estelle Troadec, ${ }^{1,2}$ Cesar Terrazas, ${ }^{5}$ Abhay R. Satoskar, ${ }^{5}$ Emine Gulsen Gunes, ${ }^{1}$ Ada Dona, ${ }^{1}$ \\ James F. Sanchez, ${ }^{1}$ P. Leif Bergsagel, ${ }^{6}$ Marta Chesi, ${ }^{6}$ Alex Pozhitkov, ${ }^{7}$ Steven Rosen, ${ }^{1}$ Guido Marcucci, ${ }^{2,8}$ \\ Jonathan J. Keats, ${ }^{1,9}$ Craig C. Hofmeister, ${ }^{10}$ Amrita Krishnan, ${ }^{1}$ Enrico Caserta, ${ }^{1,2}$ and Flavia Pichiorri ${ }^{1,2}$ \\ 1Judy and Bernard Briskin Center for Multiple Myeloma Research, Hematologic Malignancies and Stem Cell Transplantation \\ Institute, City of Hope Medical Center, Duarte, California, USA. 'Department of Hematologic Malignancies Translational \\ Science, Beckman Research Institute, City of Hope Medical Center, Duarte, California, USA. ${ }^{3}$ Department of Radiation \\ Oncology, The Ohio State University, Columbus, Ohio, USA. ${ }^{4}$ Faculty of Biology, Medicine and Health, School of Medical \\ Science, Division of Cancer Science, University of Manchester, Manchester, United Kingdom. ${ }^{5}$ Division of Experimental \\ Pathology, Department of Microbiology, The Ohio State University Medical Center, Columbus, Ohio, USA. ${ }^{6}$ Department \\ of Medicine, Mayo Clinic Arizona, Scottsdale, Arizona, USA. 'Department of Computational and Quantitative Medicine, \\ Beckman Research Institute, City of Hope, Duarte, California, USA. ${ }^{8}$ Gehr Family Center for Leukemia Research, \\ Hematologic Malignancies and Stem Cell Transplantation Institute, City of Hope, Duarte, California, USA. ${ }^{9}$ Translational \\ Genomics Research Institute, Phoenix, Arizona, USA. ${ }^{10}$ Department of Hematology and Medical Oncology, Emory \\ University School of Medicine, Atlanta, Georgia, USA.
}

High levels of circulating miR-16 in the serum of multiple myeloma (MM) patients are independently associated with longer survival. Although the tumor suppressor function of intracellular miR-16 in MM plasma cells (PCs) has been elucidated, its extracellular role in maintaining a nonsupportive cancer microenvironment has not been fully explored. Here, we show that miR-16 is abundantly released by MM cells through extracellular vesicles (EVs) and that differences in its intracellular expression as associated with chromosome 13 deletion (Del13) are correlated to extracellular miR-16 levels. We also demonstrate that EVs isolated from MM patients and from the conditioned media of MM-PCs carrying Del13 more strongly differentiate circulating monocytes to M2-tumor supportive macrophages (TAMs), compared with MM-PCs without this chromosomal aberration. Mechanistically, our data show that miR-16 directly targets the IKK $\alpha / \beta$ complex of the NF-KB canonical pathway, which is critical not only in supporting MM cell growth, but also in polarizing macrophages toward an M2 phenotype. By using a miR-15a-16-1-KO mouse model, we found that loss of the miR-16 cluster supports polarization to M2 macrophages. Finally, we demonstrate the therapeutic benefit of miR-16 overexpression in potentiating the anti-MM activity by a proteasome inhibitor in the presence of MM-resident bone marrow TAM.

Authorship note: JK and JG contributed equally to this work.

Conflict of interest: The authors have declared that no conflict of interest exists.

Copyright: (c) 2019, American Society for Clinical Investigation.

Submitted: April 5, 2019

Accepted: October 2, 2019

Published: October 8, 2019

Reference information: JCI Insight 2019;4(21):e129348

https://doi.org/10.1172/jci.

insight.129348.

\section{Introduction}

Multiple myeloma (MM) is the second most frequent blood cancer in the USA after non-Hodgkin lymphoma (1) and is characterized by the accumulation of over $10 \%$ of plasma cells (PCs) in the bone marrow (BM) (2, 3). In all cases, MM is preceded by a premalignant disease known as monoclonal gammopathy of undetermined significance (MGUS) (4-7), and - independently of specific genetic and epigenetic changes - all PCs at the beginning of the disease are dependent on the BM niche (8), which plays a pivotal role in the regulation of their growth, survival, and eventual drug resistance $(9,10)$. Of all the different essential survival signals activated in PCs by external BM stimulations, the NF-kB pathway and subsequent production of supportive growth factors (e.g., IL-6, IL-8, TNF- $\alpha$, and VEGF) and adhesion molecules, including CD44, CD184, and ICAM-1, play central roles in PC proliferation and survival and in subsequent drug resistance and/or resis- 
tance to standard-of-care proteasome inhibitors (PIs) (11). NF- $\mathrm{kB}$ constitutive activity is found in the cancer cells of MGUS and MM patients (12), and although the presence of promiscuous genetic mutations in the NF- $\kappa B$ pathway can explain NF- $\kappa B$ activation in a subset of MM patients (20\%) (13), it cannot account for the majority of the MM cases with no genetic abnormalities $(12,14)$. The long arm of chromosome 13 (13q) is variably deleted (Del. 13) in regions, which includes deletions in the region of the miR-16-15a cluster (15). Del13 occurs in almost $50 \%$ of newly diagnosed MM patients (16) and can reach up to $80 \%$ in patients with active disease (17), but it is also present at lower percentages in patients with MGUS (18). However, Del13 was seen to be correlated with the transformation of MGUS to MM, and it is found at a much higher frequency in patients with high-risk features such as $\mathrm{t}(4 ; 14)(88 \%)$ and MAF $(73 \%)$ translocations (19), highlighting that progression of $13 q$ abnormalities requires clonal evolution (20). The miR-15a/16-1 cluster is located in the intronic region of the deleted in lymphocytic leukemia 2 (DLEU2) gene coding for mature miRNAs (i.e., miR-15a and miR-16-1), which has been shown to regulate the expression of pivotal oncogenes in cancer initiation and progression, including BCL-2, MCL-1, and cyclin D (21-23). Recently, the possible role of this class of miRNAs in regulating not only the cancer cell intracellular landscape, but also the tumor microenvironment by their secretion through extracellular vesicles (EVs) has been proposed $(24,25)$. We previously showed that extracellular miR-16 is among the few microRNAs that can be used as a prognostic biomarker in newly diagnosed MM patients treated with PI-based therapy (26). Previous studies have shown the role of endogenous miR-16 in shifting macrophage $(M \Phi)$ polarization from a tumorigenic (tumor supportive $\mathrm{M} 2$ ) to tumoricidal (antitumor M1) phenotype (27). Recently published data have revealed that, in MM, tumor-associated $\mathrm{M} \Phi$ (TAMs) are recruited into the BM and protect cancer cells from chemotherapy-based apoptosis (28), and increased levels of BM TAM in MM patients are associated with poor prognosis (29).

In this study, we investigate the possible consequences of loss of miR-16 associated with Del13 in supporting PC degeneration during disease development. Specifically, we explore whether loss of miR-16 in the PCs can affect their fitness not only by changing the oncogenic landscape intracellularly, but also by affecting the tumor microenvironment.

\section{Results}

EVs and intracellular miR-16 levels are correlated. We previously reported that extracellular levels of miR16 were significantly downregulated $(P=0.003)$ in the serum of MM patients carrying Del13 in their MM cells compared with levels in patients in whom Del13 was not present (26), supporting the idea that extracellular miR-16 levels may reflect the levels of miR-16 in cancer cells. We then performed miRNA profiling of 4 different Del13 MM cell lines (RPMI-8226, U266, MM.1R, NCI-H929) and their derived EVs (Figure 1A and Supplemental Table 1; supplemental material available online with this article; https://doi.org/10.1172/jci.insight.129348DS1). miRNA analysis by Nanostring technology showed that miR-16 was more enriched in the EVs compared with its endogenous levels (Figure 1B). Conversely, the same magnitude of EV enrichment was not found for other miRNAs, including the highly endogenously expressed miR-142-3p (Figure 1, B and C), as well as miR-9, the highly expressed and well known cancer-associated biomarker released in EVs (Figure 1C and refs. 30, 31). EV miR-16 enrichment was not only observed in MM cells but, as expected, was also observed in the EV isolated from healthy BM stromal cells (Figure 1D), aligning with previously published data that show that miR$15 \mathrm{a}$ is highly released by normal stromal cells (24). We then decided to investigate whether differences in chromosome 13 status could reflect changes in miR-16 extracellular enrichment, as supported by our previously published study of MM patients (26). As expected, MM cell lines carrying Del13 (OPM2, LP-1, L363, U266, MM.1S, NCI-H929, RPMI-8226) have lower extracellular miR-16 compared with that in the few MM cell lines carrying both 13q alleles (WT) (OCIMY-5, OCI-MY1, MMM.1) (http:// www.keatslab.org) (Figure 1E).

MiR-16 is downregulated in the BM-MФ of MM patients. Because miR-16 has been shown to exert its antiMM activity in the context of the BM microenvironment (BM-ME) (32), and since PCs at the beginning of the disease rely on exocrine IL-6 production from the BM-ME for their survival (33), we investigated which cellular population is the main IL- 6 producer in this setting. Cytokine arrays show that the BM CD14 ${ }^{+}$fractions from MM patients, which we found to be mainly enriched in MФ (CD14 $\left.{ }^{+} / \mathrm{CD}_{6} 8^{+}, \mathrm{BM}-\mathrm{M} \Phi\right)$ (Supplemental Figure 1), are the main producers of NF- $\mathrm{BB}-$ dependent cytokines/chemokines, including IL-6, IL-8, and TNF- $\alpha$ (Figure 2A), a group of cytokines that were significantly upregulated in the BM-MФ of MM patients $(n=4)$ as compared with those in cancer-free donors $(n=4$, healthy donor [HD]) (Figure $2 \mathrm{~B})$. 

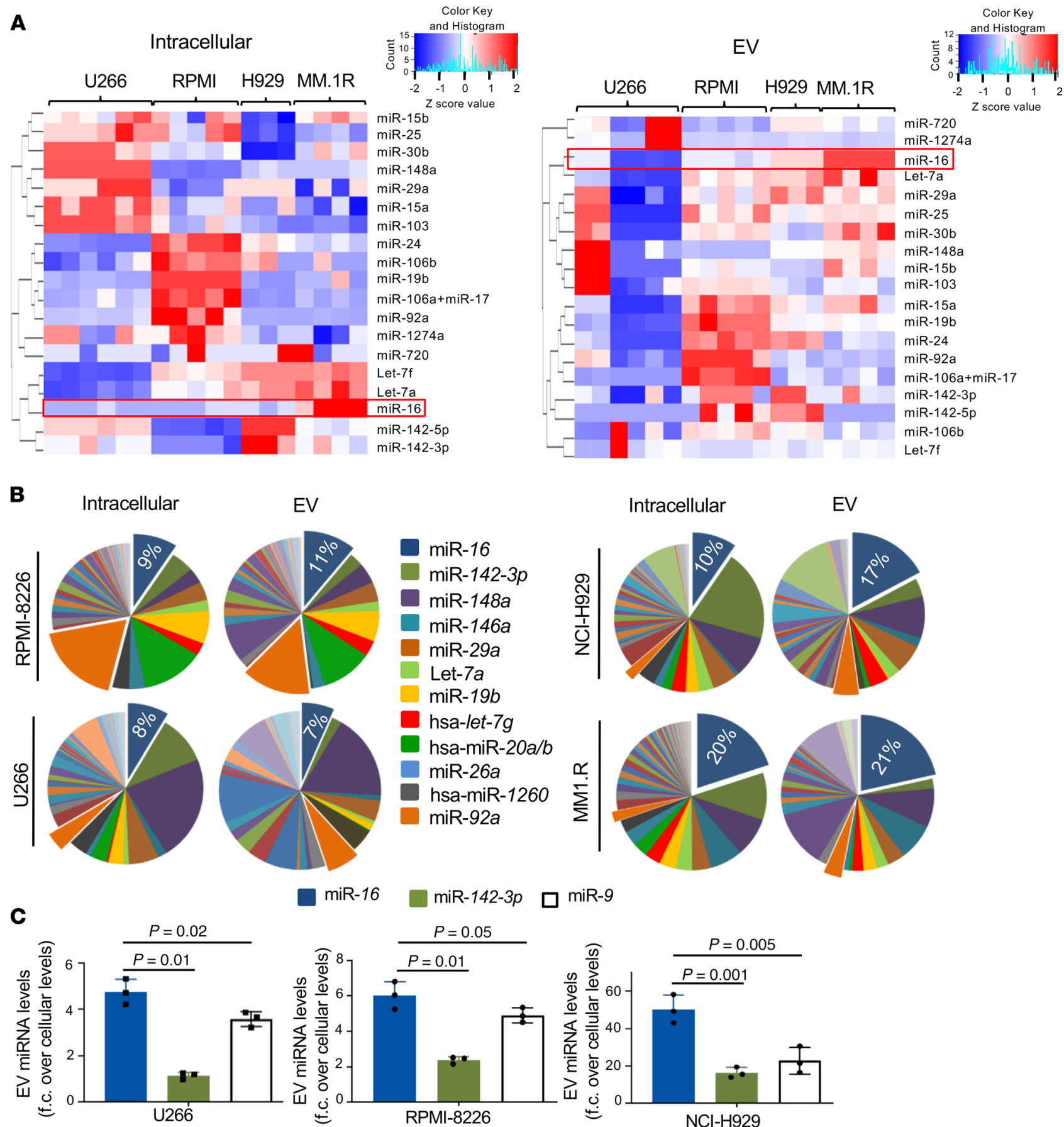

EV

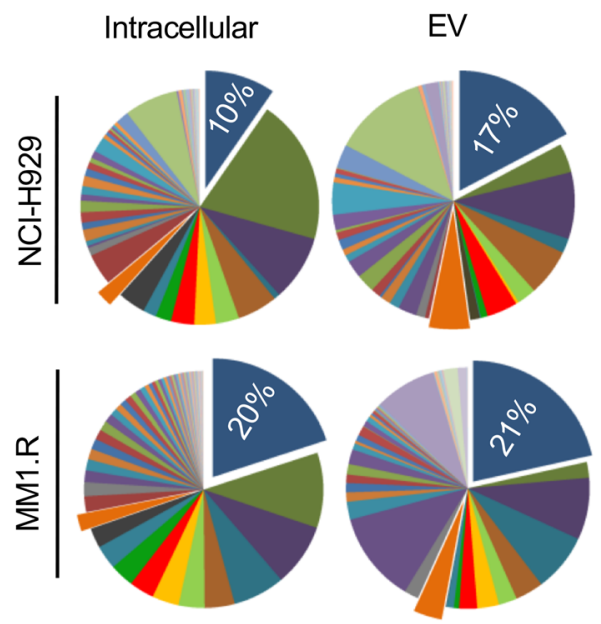

miR-16 miR-142-3p $\square$ miR-9

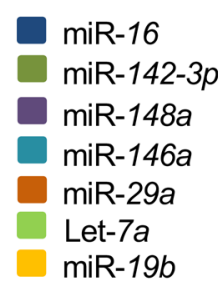

hsa-let-7g

hsa-miR-20a/b

miR-26a

hsa-miR-1260

miR-92a
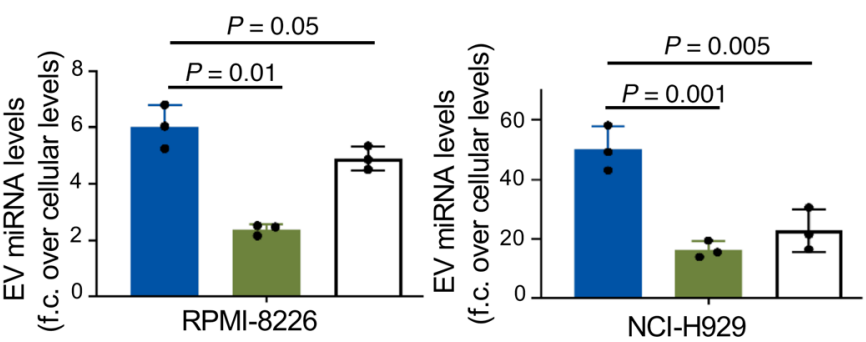

D

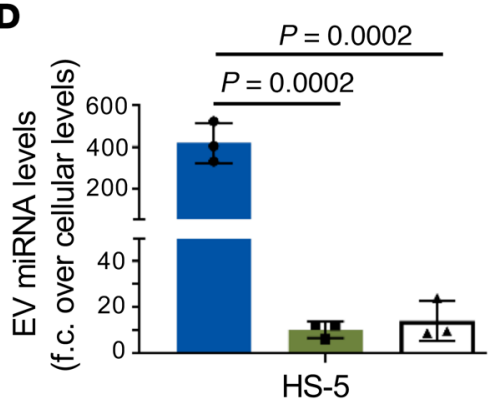

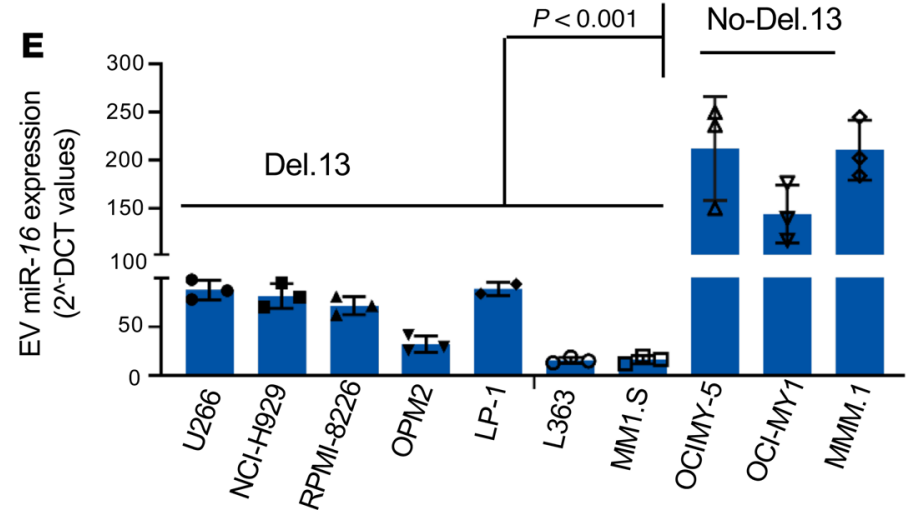


Figure 1. EVs and intracellular miR-16 levels are correlated. (A) Heatmaps showing microRNA expression profile as measured by the NanoString nCounter System in MM cells (RPMI-8226, U266, NCI-H929, MM1.R) (left panel) and in extracellular vesicles (EV) secreted by those cells (right panel). Each column represents 1 sample/cell line with red representing upregulated and blue representing downregulated. Each cell line was run at least in triplicate. Heatmaps were performed using the G-plots package heatmap.2 program, and colored scales were generated using the $Z$ score values. (B) Pie charts showing the percent of the 59 highest intracellular microRNA expression levels and their corresponding EV secreted levels in the 4 cell lines tested. The 12 highest microRNA expression levels among cell lines from miR-16 (blue) to miR-92a (orange) are highlighted in a colored spectrum. (C) miR-16, miR-142-3p, and miR-9 expression levels in EVs released by U266, RPMI-8226, and NCI-H929 MM cell lines. Data are presented as fold change (f.c.) over intracellular microRNA expression for each miRNA. (D) Parallel to $\mathbf{C}$ using HS-5 cell line. Values represent the mean \pm SD; $P$ values were calculated using ordinary 1-way ANOVA multicomparisons test. Each experiment was performed in triplicate. (E) qPCR showing miR-16 expression in EVs released by Del13 MM cell lines (U266, NCI-H929, RPMI-8226, OPM2, LP-1, L363, MM.1S) and non-Del13 MM cell lines (OCIMY-5, OCIMY-I, MMM.1). Data are presented as $2^{-\triangle C T}$ values. Values represent the mean \pm SD; $P$ values were calculated using 2-tailed unpaired $t$ test. Each experiment was performed in triplicate; the obtained $P$ values are reported.

We then asked whether this population could be affected by differential expression of EV miR-16. Our data show that miR-16 is significantly downregulated in the MM-BM-MФ $(n=7 \mathrm{pts})$ compared with that isolated from healthy donors ( $n=7 \mathrm{pts}$ ) (Figure 2C). To unequivocally evaluate the downregulation of miR16 in the BM compartment of MM patients, we also compared the miR-16 levels between MM-BM-M $\Phi$ and their matched peripheral blood monocytes (PB-M) ( $n=3$ patients). We found a significant decrease in miR-16 expression in MM-BM-MФ as compared with their circulating fraction (Figure 2D).

To further assess whether miR-16 downmodulation may be associated with monocyte to $M \Phi$ differentiation toward the M2-MФ phenotype, we first induced differentiation of PB-M isolated from MM patients in vitro in the presence of the M2 differentiation factor MФ CSF (M-CSF) for 7 days. As expected, upon M-CSF treatment, the cells appeared more filamentous, having an endothelial-like shape indicative of the M2 phenotype, as compared with the control untreated undifferentiated (UI) cells (Figure 2E). A GFP fluorescence latex beads-phagocytosis assay showed that cells treated with M-CSF were fully differentiated $\left(84 \% \mathrm{GFP}^{+}\right)$as compared with UI cells $\left(4.63 \% \mathrm{GFP}^{+}\right)$(Figure $\left.2 \mathrm{~F}\right)$ and showed significant increases in the mRNA expression of typical M2-MФ expression markers, including CD163 and IRF4 (Figure 2, G and H). We also observed that M2-M $\Phi$ had a significant decrease in miR-16 expression upon differentiation (Figure 2I). In summary, our findings show that miR-16 is downmodulated in the MM-BM-M $\Phi$, and its downregulation in vitro is associated with an $\mathrm{M} 2-\mathrm{M} \Phi$ phenotype.

Extracellular miR-16 impairs $M M-E V$-induced M2-MT. Because previously published data have shown that, by producing soluble factors, MM cells can modify the BM niche and induce $\mathrm{M} \Phi$ polarization toward an M2 tumor-supportive phenotype (34), we investigated whether the BM acellular fraction (BM-ac), the soluble portion of the BM, of MM patients could directly affect $\mathrm{M} \Phi$ differentiation. We found that monocytes isolated from MM patients (MM-PB-M) tend to differentiate to an M2-like $\mathrm{M} \Phi$ when treated with their matched BM-ac for 7 days (Figure 3A). Specifically, we observed that, after 7 days of incubation, the shape of PB-M was filamentous and appeared similar to those differentiated with M-CSF (Figure 3A and Figure 2E). miR-16 expression was markedly downregulated in the PB-M differentiated with the BM-ac compared with that in the matched UI PB-M. miR-16 levels upon in vitro differentiation were fully comparable with those in MM-BM-M $\Phi$ isolated from the same patient (Figure 3B). We then sought to identity the component in the BM-ac that was responsible for inducing an M2-like phenotype. Our data show that EVs isolated from the BM-ac of $4 \mathrm{MM}$ patients induced differentiation of PB-M isolated from healthy donors to M2-M $(n=4)$, whereas no differentiation was observed when cells were incubated with the EV-depleted fractions (Figure 3C and Supplemental Figure 2A). Our data show that these differentiated cells are effectively $\mathrm{M} \Phi$, as they engulfed and digested fluorescent latex beads, as demonstrated by a phagocytosis assay (Figure 3D). Interestingly, PB-M did not show any differentiation toward an M2 phenotype, as assessed by the M2 markers CD163 and CD206, when treated with the conditioned media of the cultured total BM cellular population isolated from 3 cancer-free donors (BM-HD), as compared with those isolated from MM patients. In fact, almost all of the cells were dead after 7 days of treatment with the conditioned media of BM-HD (Supplemental Figure 2B). To assess whether the EVs produced by MM cells are directly responsible for driving PB-M differentiation to M2-MФ, we isolated EVs from the conditioned media of Del13 MM cells (NCI-H929 and MM.1S). We found that healthy PB-M treated with MM-derived EVs (MM-EVs) for 7 days tend to differentiate toward an M2 phenotype, as shown by the increased expression of the M2 marker CD163 (Figure 3E). We have previously shown that newly diagnosed MM patients with Del13 have lower circulating miR-16 levels compared with those in patients with non-Del13 (26), and other groups have found 

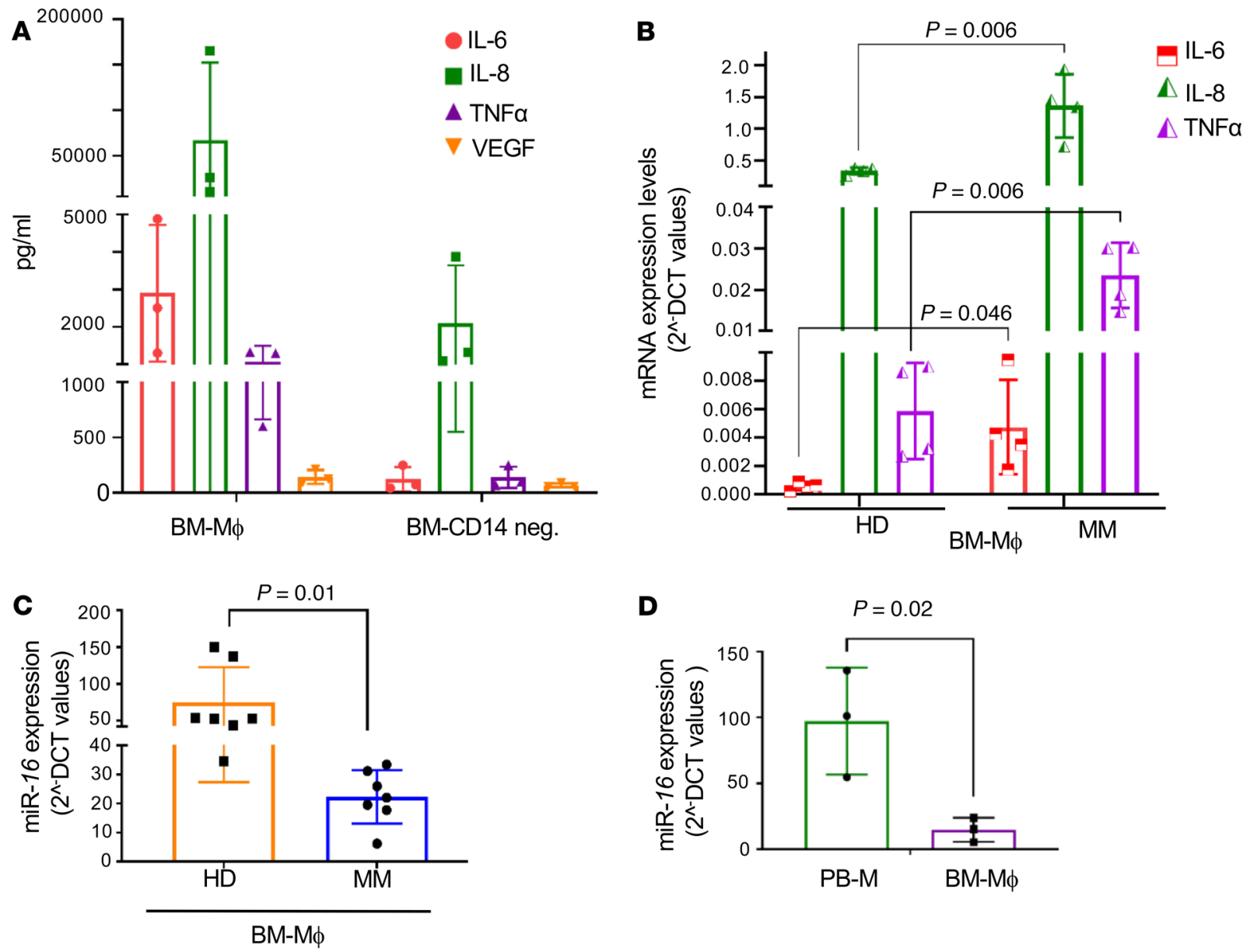

E
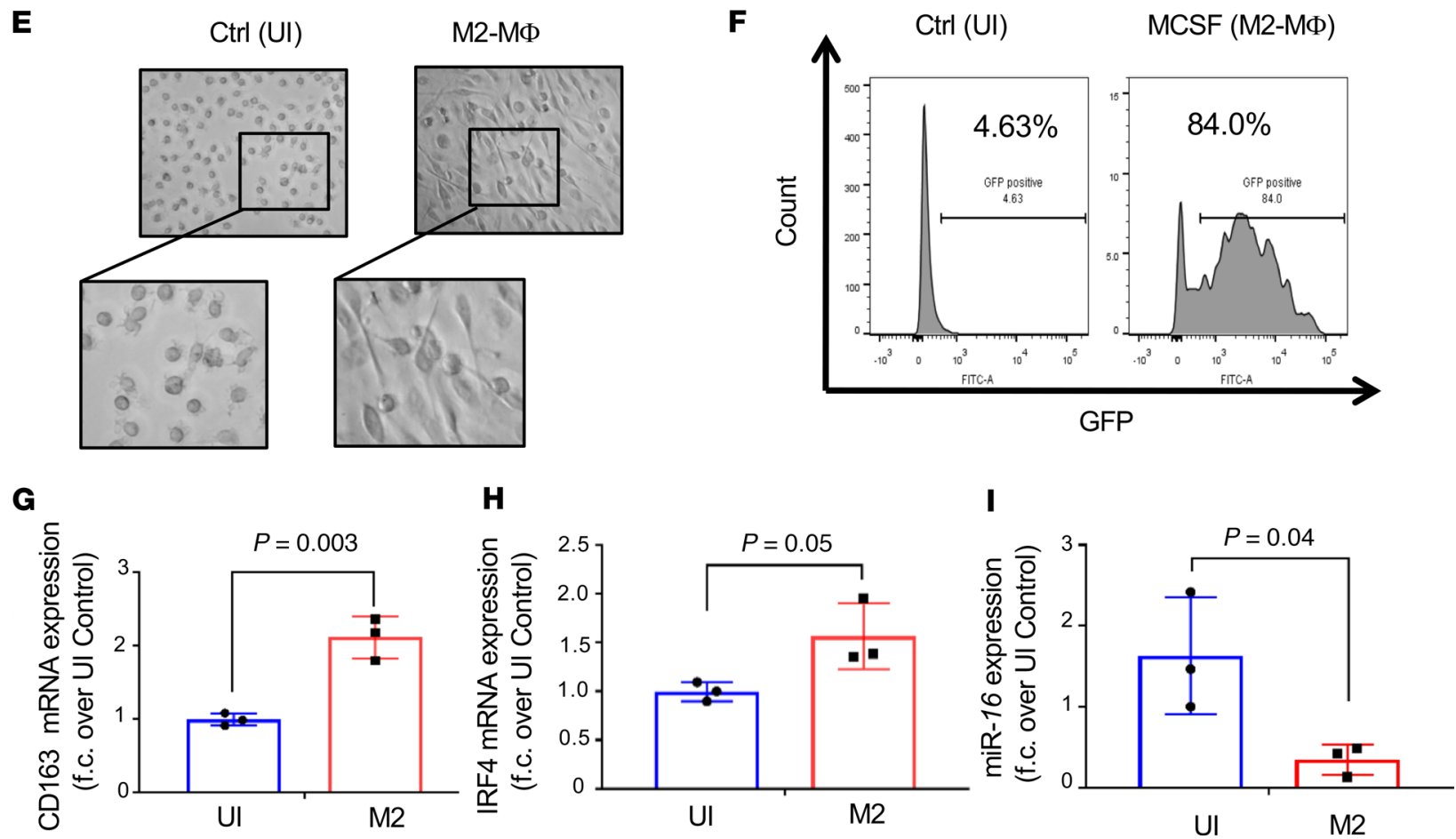
Figure 2. MiR-16 is downregulated in the BM-MФ of MM patients (A) Cytokine array showing, under stimulated conditions (i.e., in the presence of single-stranded RNA-mir-25 (ssRNA-miR-25), which stimulates TLR-7 and -8, the levels of NF-kB-induced, M2-associated cytokines (IL-6, IL-8, TNF- $\alpha$, and VEGF) secreted by CD14+ macrophages (BM-MФ), and CD14- cells (BM-CD14 neg.) isolated from the BM of MM patients $(n=3)$. Data represent mean values \pm SD. Cells were incubated with ssRNA-miR-25 for 24 hours prior to detection of cytokines. Cytokine levels were measured in pg/mL using a multiplex cytokine assay. (B) qPCR showing mRNA expression levels of IL-6, IL-8, and TNF- $\alpha$ in BM-M $\Phi$ isolated from MM patients (MM) or cancer-free donors (HD) ( $n=4$ /group). $P$ values were calculated using 2-tailed multiple $t$ test. (C) qPCR showing decreased miR-16 expression in BM-M $\Phi$ isolated from MM patients as compared with that isolated from HD ( $n=7 /$ group). (D) Real-time PCR showing decreased expression of miR-16 in BM-M compared with that in monocytes isolated from the peripheral blood (PB-M) of the same MM patients $(n=3)$. Data are presented as $2^{-\Delta C T}$ values. (E) Representative images captured by light microscopy showing PB-M differentiated to M2-like MФ (M2-MФ) with macrophage CSF (M-CSF) treatment for 7 days (right panel) as compared with undifferentiated PB-M (UI) (left panel). Magnification ×40. (F) Flow cytometric analysis showing phagocytosis of M2-M $\Phi$ in vitro. Phagocytosis assay was performed using latex beads coated with GFP fluorescently labeled IgG antibody. The engulfed fluorescent beads were detected by flow cytometry. Differentiated PB-M to M2-MФ in the presence of M-CSF showed $84 \%$ GFP+ cells in contrast to UI cells that were only $4.63 \%$. (G-I) Real-time PCR showing increases in CD163 (C) and IRF4 (H) mRNA expression, as well as a decrease in miR-16 expression (I) in M2-MФ as compared with UI. CD163 and IRF4 levels were presented as fold changes over UI controls. miR-16 levels were presented as $2^{-\Delta C T}$ values ( $n=3$ patients). Data reported in C, D, and G-I represent the mean $\pm \mathrm{SD} ; P$ values were calculated using a 2-tailed unpaired $t$ test.

that increased levels of the EV miR-15a (miR-15a/16-1 cluster) can inhibit MM cell growth (24). We then decided to investigate whether high levels of ectopic extracellular miR-16 can impair monocyte to M2-MФ differentiation. When PB-M isolated from healthy donors were treated with EVs isolated from the conditioned media of either MM.1S or NCI-H929 and concomitantly incubated with a double-stranded miR-16 mimic encapsulated in lipid rafts (ds-miR-16), we observed lower CD163 surface expression as compared with that in PB-M treated with EVs in the presence of double-stranded miR-223 mimic (EV+miR-223) or scramble $(\mathrm{EV}+\mathrm{Scr})$ (Figure $3 \mathrm{~F}$ and Supplemental Figure 2C). Interestingly, a higher increase in CD163 levels was observed for both UI and differentiated PB-M when treated with a miR-223 mimic, a miRNA that is known to be implicated in maintaining $\mathrm{M} \Phi$ in an M2-like phenotype (Figure $3 \mathrm{~F}$ and Supplemental Figure 2C; ref. 35). In support of these data, the EV-treated monocytes appeared less filamentous, and few or no detectable endothelial-shaped cells were present upon treatment with double-stranded miR-16 (ds-miR-16), in contrast to that from scramble sequences (Figure 3G and Supplemental Figure 2D). Taken together, our data show a functional effect of miR-16 in impairing M2-M $\Phi$ differentiation.

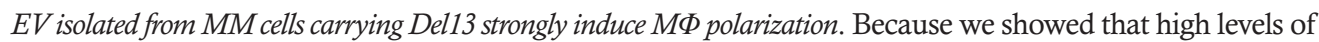
ectopic extracellular miR-16 impair monocyte to MM-EV-driven M2-MФ differentiation, we decided to investigate whether differences in miR-16 EV enrichment could differentially impact this effect. We isolated EV from MM cells carrying either a well-characterized Del13 (OPM2, LP-1, L363) or intact WT alleles (OCIMY-5, OCIMY1, MMM.1). These EVs were found to express different miR-16 copy numbers (Figure 1E). In agreement with our data, we found that EVs isolated from Del13 cell lines induced significantly higher levels of МФ differentiation to an M2-like phenotype compared with EVs isolated from cells carrying the WT allele, as assessed by levels of M2 markers such as the mannose receptor (CD206) and CD163 as early as 4 days of PB-M treatment using the same primary $\mathrm{CD} 14^{+}$cells across cell lines $(P<0.01)$ (Figure $\left.4, \mathrm{~A}-\mathrm{C}\right)$. We then investigated whether EVs from patients carrying Del13 could also induce monocyte differentiation. We found that the BM-ac isolated from patients carrying Del13 significantly induced more PB-M differentiation toward an M2 like phenotype as compared with that from patients carrying the WT form ( $n=3$ /group) (Figure 4, D-F).

Evaluation of $M \Phi$ polarization in a miR-15a/16-1-KO model. To further examine the in vivo effects of miR-16 on MФ polarization, we used a miR-15a/16-1-KO mouse model that was thoroughly established by Klein et al. (21), which resembles the human miR-15a/16-1 cluster deletion by carrying the corresponding alteration on mouse chromosome $14 \mathrm{qC} 3$. Since markers of differentiation were not observed at basal levels (data not shown; ref. 27), primary basal state spleen $\mathrm{M} \Phi(\mathrm{M} 0-\mathrm{M} \Phi)$ (Supplemental Figure 3A) were isolated from miR-15a/16-1-null or WT B6 mice and induced to differentiate to M2-like cells following M-CSF and IL-4 treatment ex vivo (27). In response to IL-4 treatment, M0-M $\Phi$ isolated from miR-15a/16-1-null mice presented a significantly more pronounced M2 phenotype compared with that in the WT mice, as measured by the expression of mouse M2 markers such as CD206 and C-type lectin (Dectin-1) at an early (48 hours) (Figure 5, A, C, E, and G) or late (6 days) differentiation time (Figure 5, $\mathrm{B}, \mathrm{D}, \mathrm{F}$, and $\mathrm{H})$. As early as 48 hours, miR-15a/16-1-null $\mathrm{M} \Phi$ expressed an average of a 10-fold increase $(6.25 \% \pm 1.6 \%)$ in CD206 (Figure 5, A and C) and an almost 5-fold increase (23.5\% $\pm 8 \%$ ) in Dectin-1 (Figure 5, E and G) as compared with levels from WT mice, which were $0.6 \% \pm 0.4 \%$ and $4.9 \% \pm 0.7 \%$, respectively $(P=0.001, \mathrm{CD} 206 ; P=0.008$, Dectin- $1 ; n=4$ /group). Isotype controls for each panel of the $\mathrm{M} \Phi$ population at respective time points of 2 days (Figure 5, A and E) and 6 days (Figure 5, B and F) were 
A
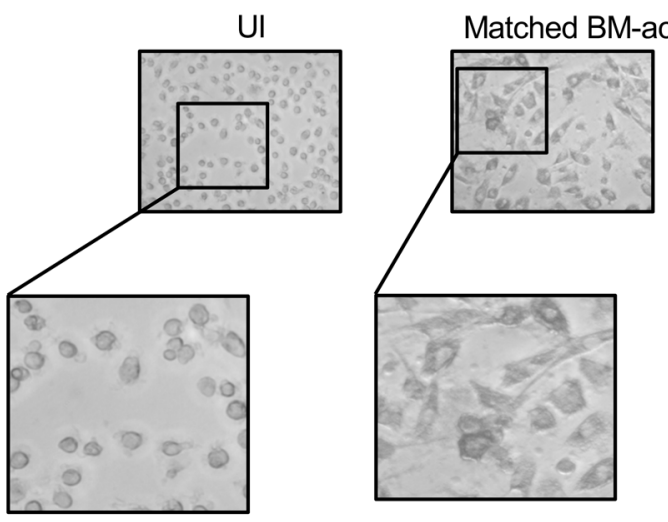

PB-M+ EV-depleted

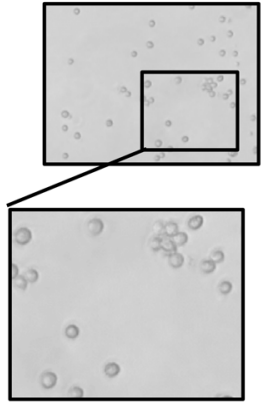

MM.1S
D EV
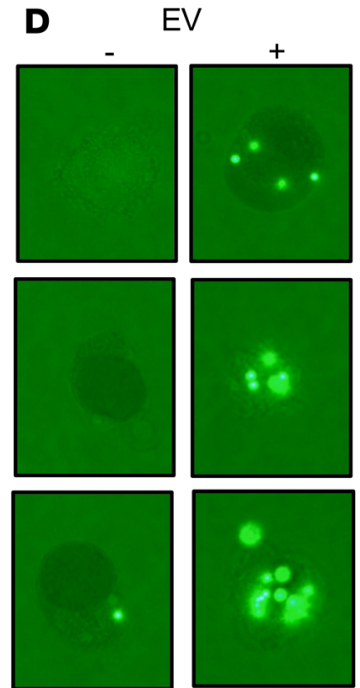

PB-M

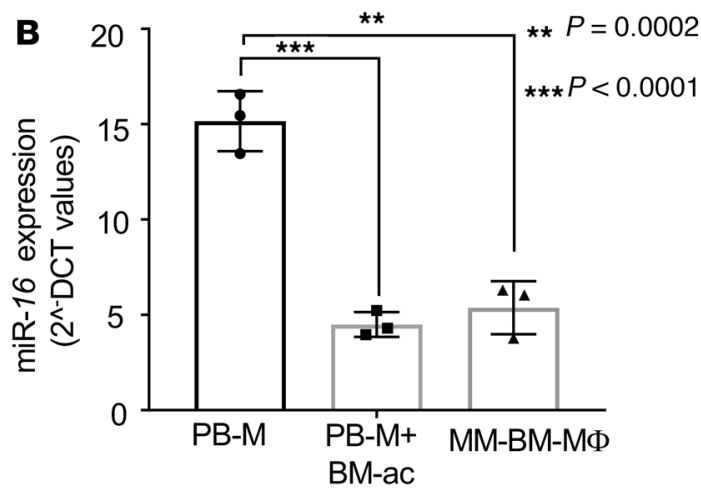

$\mathbf{F}$

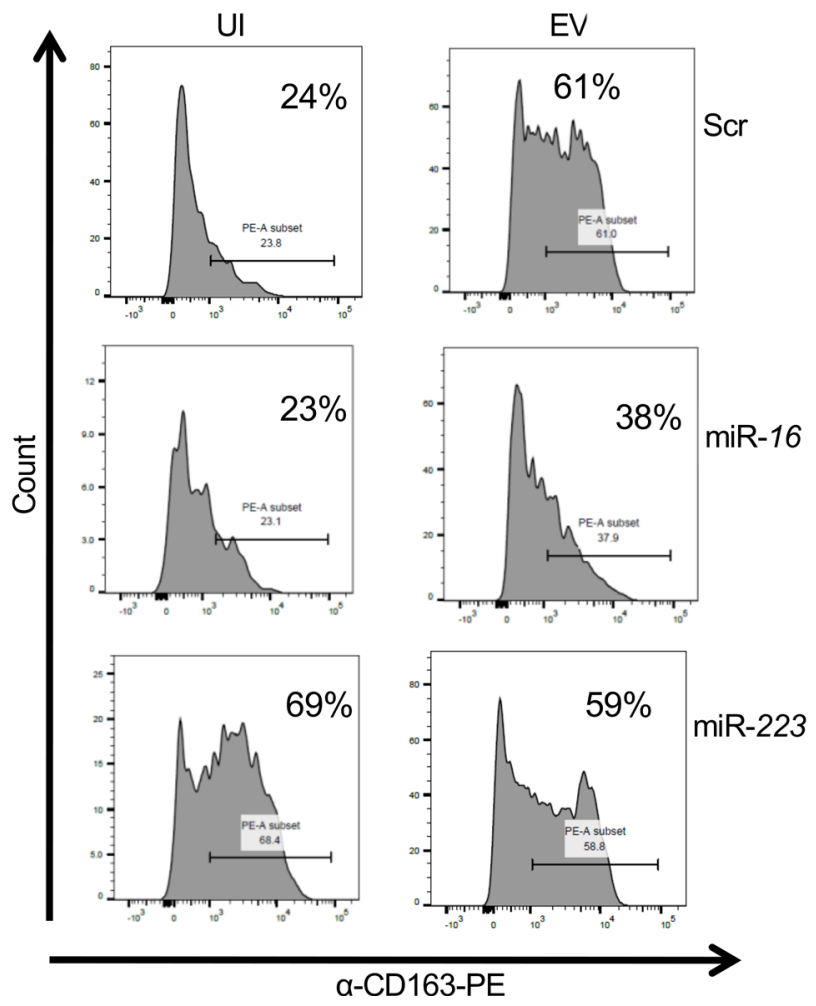

E

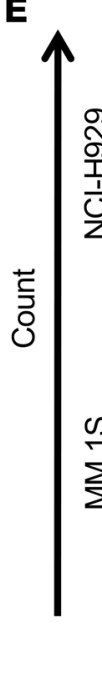

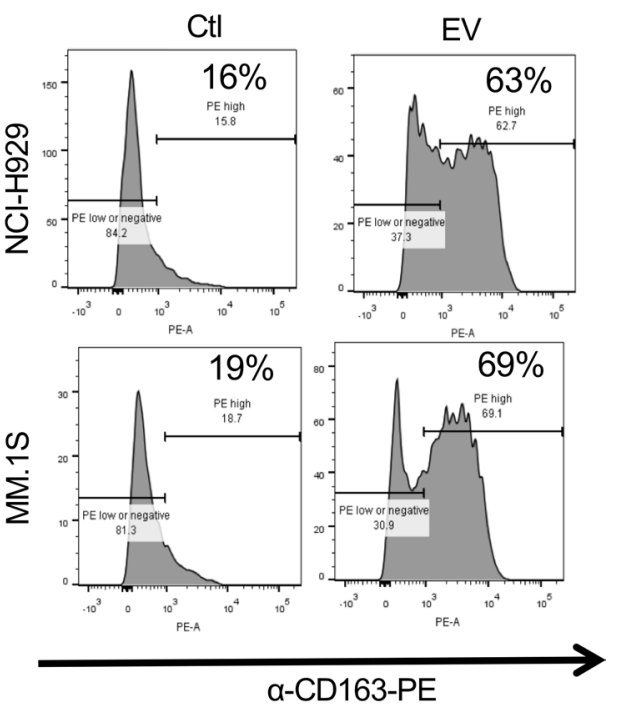

G

MM.1S
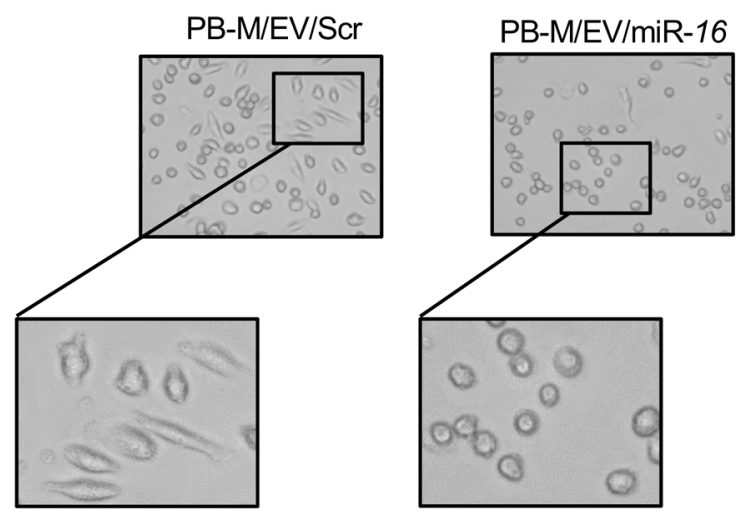
Figure 3. Extracellular miR-16 impairs MM-EV-induced M2-MФ. (A) Representative images captured by light microscopy showing PB-M isolated from a $M M$ patient differentiated to $M 2-M \Phi$ in the presence of the matched BM acellular fraction (matched BM-ac) (right panel), in contrast to undifferentiated PB-M (UI) (left panel). (B) qPCR showing decreased expression of miR-16 in PB-M isolated from MM patient and differentiated to M2-MФ in the presence of the matched $B M$ acellular fraction (PB-M + BM-ac), as well as in total $M \Phi$ isolated from the $B M$ of the same patient (MM-BM-M $\Phi$ ), as compared with undifferentiated PB-M using samples obtained from $n=3$ MM patients. Values represent the mean \pm SD; $P$ values were calculated using ordinary 1-way ANOVA multicomparison. (C) Representative images captured by light microscopy showing differentiation of PB-M obtained from a HD incubated with EV isolated from the BM-acellular fraction of a MM patient (BM-ac) (left panel) or EV-depleted BM-ac (right panel) ( $n=4$ patients; see Supplemental Figure 2B). (D) Representative images showing phagocytosis of latex beads coated with GFP fluorescently labeled lgG antibody by PB-M differentiated to M2-M $\Phi$ when incubated with EV isolated from the BM-ac of a MM patient (+) (right panel), whereas no phagocytosis was observed when PB-M were incubated with the EV-depleted BM-ac (-) (left panel). (E) Flow cytometric analysis showing percent increase in expression of M2-MФ surface marker (CD163) on PB-M treated with EV isolated from the conditioned media of a MM cell line, $\mathrm{NCl}-\mathrm{H} 929$, for 7 days (EV) (upper right panel) and compared with cells incubated with EV-depleted acellular fraction (CtI) (upper left panel). The same effect was seen using EV isolated from another MM cell line (MM.1S) (lower panel). Gating strategy was set using IgC anti-PE antibody isotype control. (F) Flow cytometric analysis showing percent surface expression of CD163 on PB-M differentiated with EV isolated from the conditioned media of MM.1S cells and concomitantly incubated with either ds-miR-16 (middle right panel), ds-miR-223 (lower right panel), or scramble control (Scr) (upper right panel). CD163 percent surface expression on undifferentiated cells (UI) incubated with the microRNAs cited above are also shown (left panels). Gating strategy was set using IgG anti-PE antibody isotype control. (C) Representative images captured by light microscopy showing impairment of PB-M differentiation to M2-M $\Phi$ in the presence of ds-miR-16. PB-M were incubated with the EV isolated from MM.1S, along with ds miR-16 (right panel) or Scr control (left panel) for 7 days. All magnifications $\times 40$.

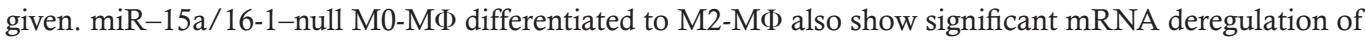
M2-effector functional markers such as IL10 ${ }^{\text {hi }}$ and IL12 $2^{\text {lo }}$ (Supplemental Figure 3, B and C).

$M i R$-16 directly regulates the expression of $I K K \alpha / \beta$ complex. We have shown that cytokines and growth factors - direct targets of the NF- $\mathrm{KB}$ canonical pathway, which is dependent on the IKK $\alpha / \beta$ kinase complex (36) are the main players secreted by MM-BM-MФ. Previous studies have also shown that the canonical (classical) $\mathrm{NF}-\mathrm{kB}$ pathway is an important regulator of the TAM transcriptional program (37), whereas the noncanonical pathway is mainly involved in immune cell differentiation and organogenesis (38). We then proceeded to investigate whether ectopic expression of miR-16 could regulate the IKK complex in primary samples. Our data show that, when $\mathrm{M} \Phi$ isolated from MM patients were treated with ds-miR-16, the expression levels of both IKK $\alpha$ and $-\beta$ were significantly downmodulated, in contrast to treatment with Scr control (Figure 6A). The same effect was seen when a МФ-like malignant cell line, U-937, or a human stromal cell line, HS-5, were transfected with ds-miR-16 (Figure 6, B and C). We also found that MФ isolated from miR-15a/16-1-KO mice had significantly higher endogenous IKK $\alpha / \beta$ expression levels as compared with WT M $\Phi$ both at the protein and mRNA levels (Figure 6, D-F, and Supplemental Figure 4A). Interestingly, given the importance of the IKK $\beta$ subunit in signaling the NF- $\mathrm{\kappa B}$ canonical pathway (36), differentiation of PB-M to M2-like MФ using M-CSF showed significantly higher expression of IKK $\beta$ as compared with cells differentiated to M1-like MФ (Supplemental Figure $4 \mathrm{~B})$. NF- $\mathrm{KB}$ pathway activation is not only associated with the production of MM-supportive cytokine/chemokines by the BM-ME, but it is also involved in MM cell autologous survival and proliferation (14). Because Del13 containing the miR-15a/16-1 cluster (15) is observed in a high percentage of MM patients (17), we assessed whether MM-PCs carrying this genetic abnormality could show significant changes in the mRNA expression of the IKK complex. Using the MMRF CoMMpass IA13 dataset, we modeled the occurrence of Del13 as a function of sex, age, 13q14 deletion, and expressions of ENSG00000213341 (IKKA), ENSG00000104365 (IKKB), ENSG00000231607 (DLEU2), and ENSG00000075624 (ACTB). The gene ACTB (actin) was chosen as a housekeeping gene not expected to be correlated to the Del13. We found a significant increase in IKKa mRNA expression (model coefficient $=0.13, P<0.001$ ) in the MM-PCs isolated from 355 newly diagnosed patients carrying Del13, compared with levels in 330 patients without this aberration. As expected, a strong correlation between the presence of Del13 and a decrease in miR-15a/16-1 host gene (DLEU2) mRNA expression in CD138 + MM cells carrying Del13 was also observed (model coefficient $=-0.08, P<0.001$ ). In support of these data, miR-16 ectopic expression inhibited IKK complex expression in multiple MM cell lines (MM.1S, NCI-H929, U266), and this effect was not only restricted to IKK $\alpha$, but also to IKK $\beta$ (Figure 6, G and $\mathrm{H}$ ). Because we did not find significant upregulation of IKK $\beta$ at the mRNA level in MM-PCs with Del13, we investigated whether this subunit could instead be regulated at the translational level or be a miR-16 indirect target. By using TargetScan software prediction (39), we found a highly conserved microRNA responsive sequence (MRE) in the 3'UTR of IKK $\beta$ at position 603-610. To assess whether miR-16 could affect IKK $\beta$ expression by directly binding its 3 'UTR and subsequently affect protein translation, we cloned a specific region containing nucleotides $487-736$ of the IKK $\beta$ 3'UTR that covers the predicted miR-16 targeting sequence in a commercially available luciferase construct, pGL4.11. Luciferase reporter assays showed that miR-16 significantly downregulated the activity of the reporter linked to IKK $\beta$ 3'UTR in U266 and HS-5 cell lines, compared with Scr control (Figure 
A

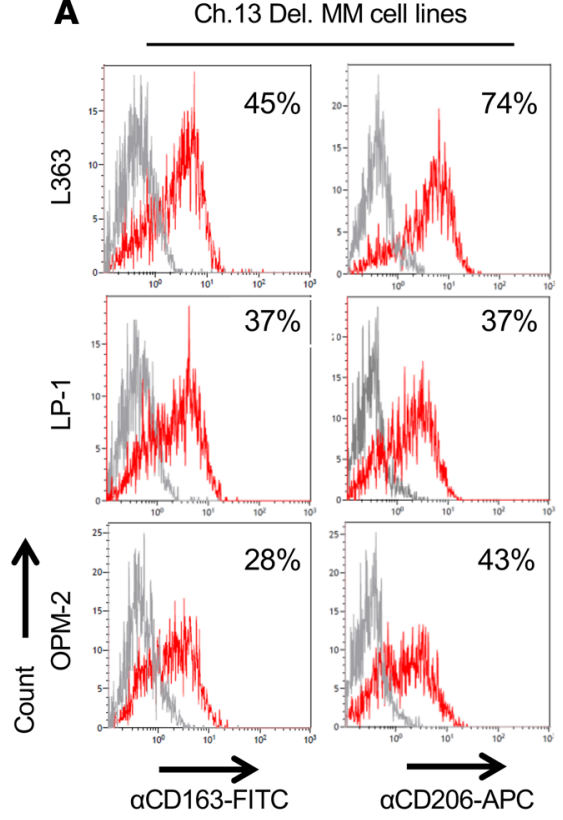

B No Del-13 MM cell lines
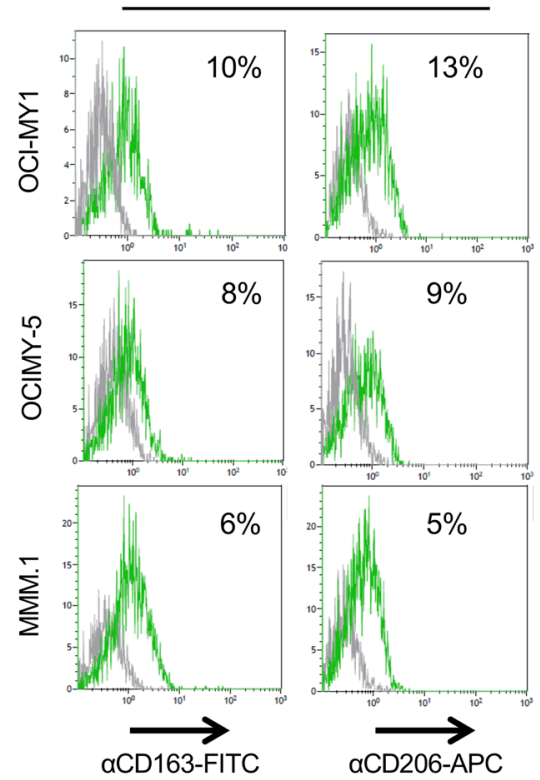

Iso-C

CD163 or CD206 Ch.13 Del cell lines CD163 or CD206 No Del-13 cell lines
D
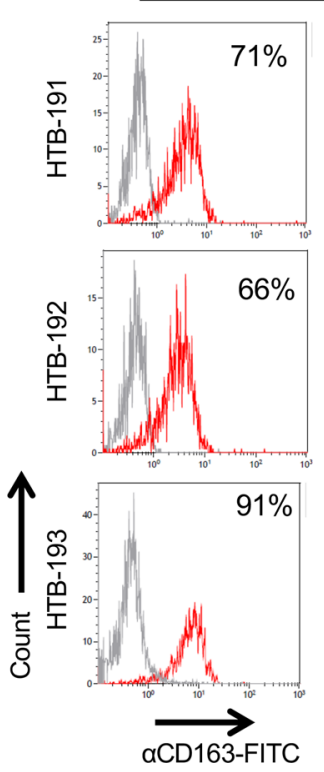

E

E $\quad$ WT Chr. $13 \mathrm{MM}$ Pts.

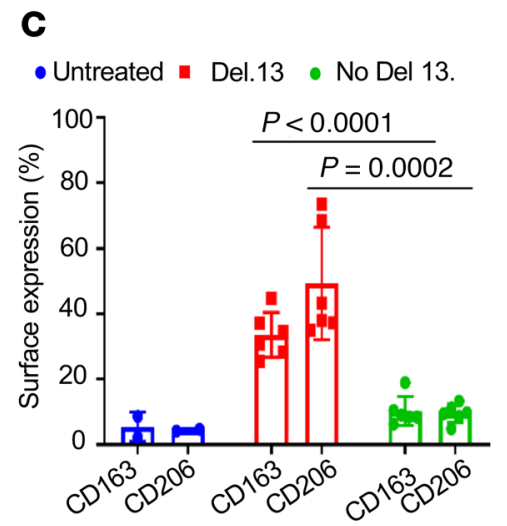

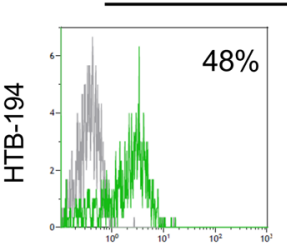
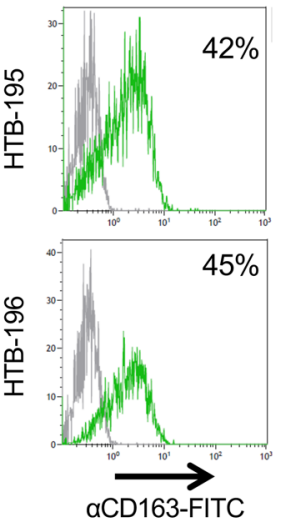

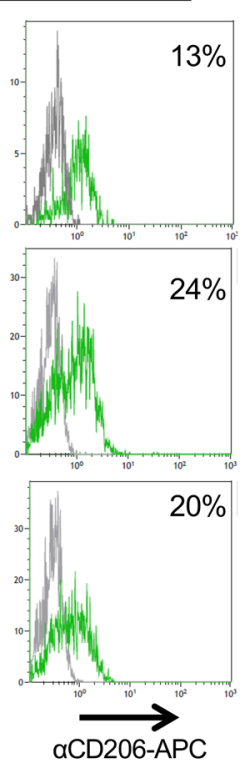

Iso-C

CD163 or CD206 Ch.13 Del patients CD163 or CD206 No Del-13 patients

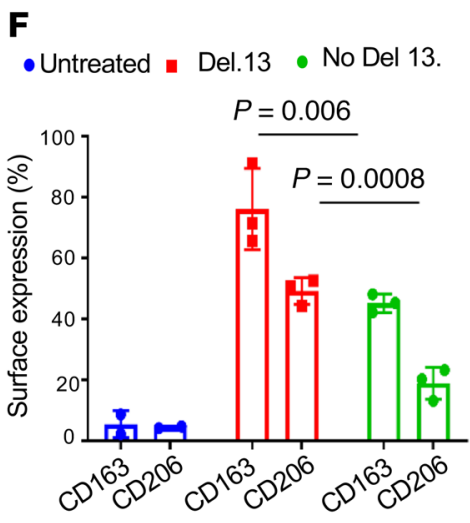

Figure 4. EV isolated from MM cells carrying Del13 strongly induce $\mathbf{M} \Phi$ polarization. (A and $\mathbf{B})$ Representative flowsight cytometric analysis overlaid with the respective isotype control (Iso-C) showing comparable CD163 and CD206 percent surface expression on differentiated PB-M isolated from a healthy donor upon treatment with the EV isolated from Del13 cell lines (L363, LP-1, OPM2) (A) or no Del13 cells (OCIMY-5, OCIMY-I, MMM.1) (B) for 4 days. Gating strategy was set using a mix of IgC anti-FITC and IgC anti-APC antibodies isotype control. (C) Bar dot plots showing average CD163 and CD206 percent surface expression on differentiated PB-M treated with EV-Del13 cell lines (red, $n=3$ ) or EV-no-Del13 cell lines (green, $n=3$ ). Each dot plot represents a flowsight cytometric analysis percent reading. Statistical comparisons for each surface marker were performed only between Del13 and no-Del13 cell lines. Values represent the mean \pm SD; $P$ values were calculated using the 2 -tailed unpaired $t$ test. Percent of untreated PB-M surface markers expression are indicated in blue and were only used as internal control. (D and $\mathbf{E}$ ) Representative flowsight cytometric analysis overlaid with the respective isotype control (Iso-C) showing comparable CD163 and CD206 percent surface expression on differentiated PB-M isolated from a healthy donor upon treatment with the BM-acellular fraction (BM-ac) of MM patients carrying Del13 (HTB-191, HTB-192, HTB-193) (D) or BM-ac of MM patients with no Del13 (HTB-194, HTB-195, HTB-196) (E) for 4 days. Gating strategy was set using a mix of IgC anti-FITC and IgC anti-APC antibody isotype controls. (F) Bar dot plots showing average CD163 and CD206 percent surface expression on differentiated PB-M treated with BM-ac of MM patients with Del13 (red, $n=3$ ) or no-Del13 (green, $n=3$ ). Percent of untreated PB-M surface markers expression are indicated in blue. Each dot plot represents a flowsight cytometric analysis percent reading. Statistical comparisons for each surface marker were performed only between Del13 and no-Del13 patients. Values represent the mean \pm SD; $P$ values were calculated using 2-tailed unpaired $t$ test. 
A

Differentiation:

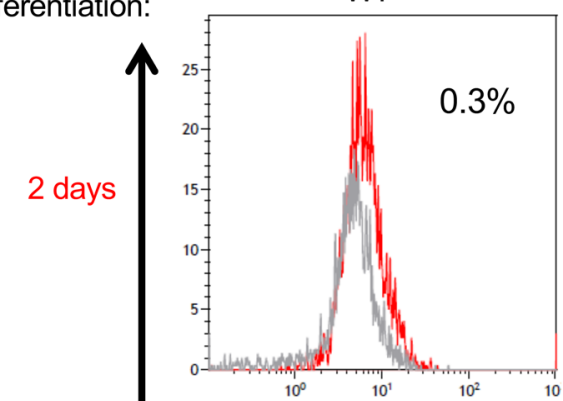

B

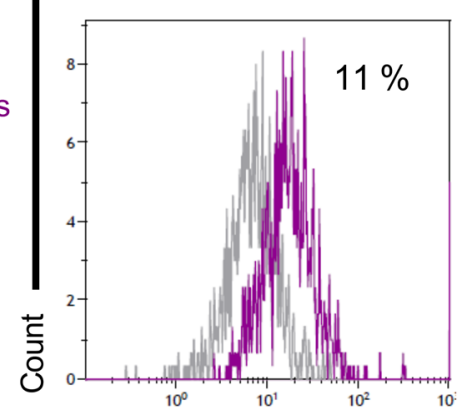

CD206-Alexa Fluor 488

Iso-C

E

Differentiation:

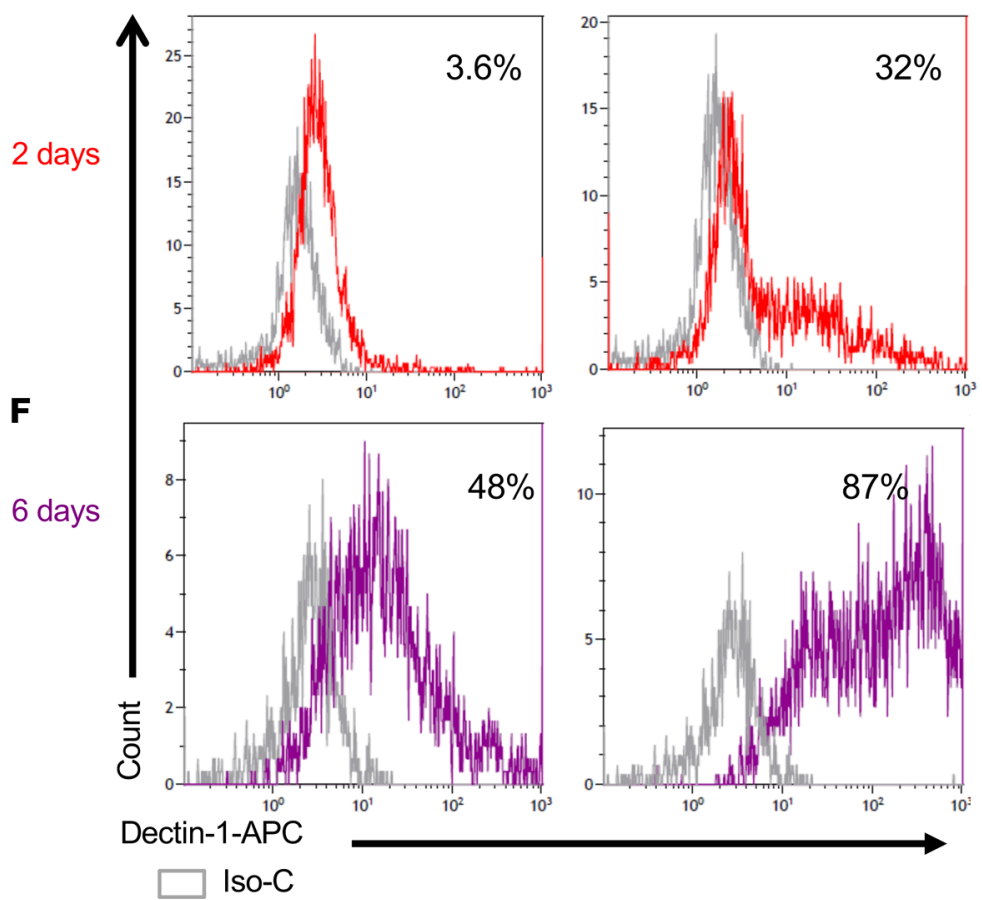

C

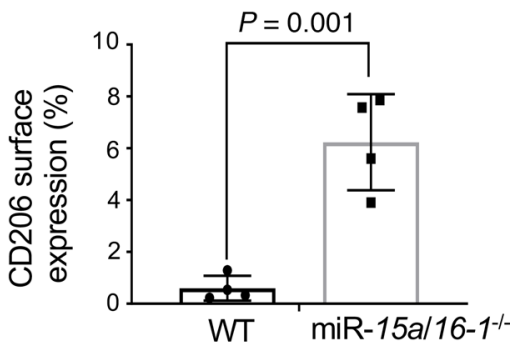

D
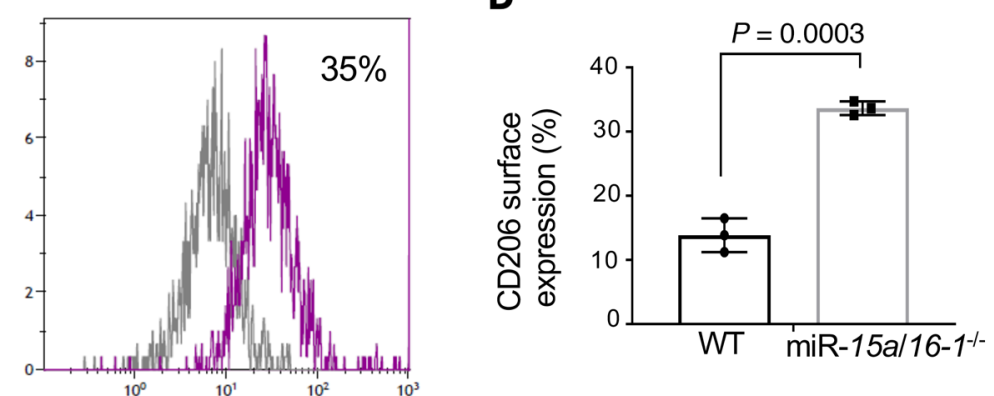

G

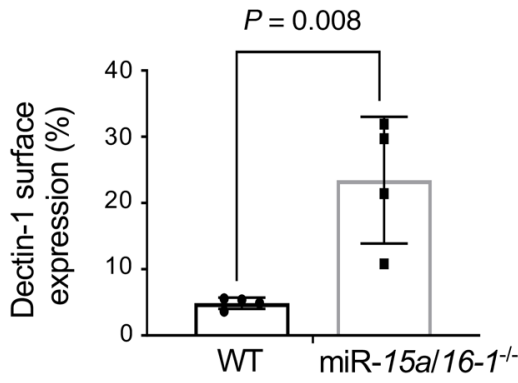

H

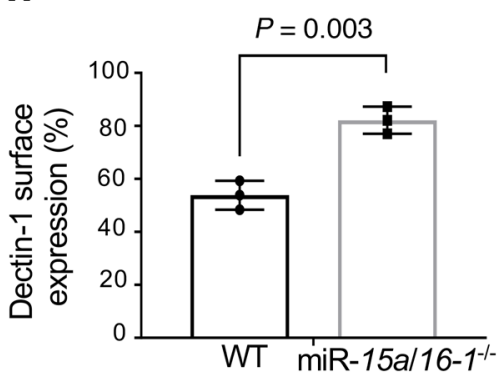

Figure 5. Evaluation of MФ polarization in a miR-15a/16-1-KO model. (A and B) Representative flowsight cytometric analysis with overlaid isotype control (Iso-C) showing comparable CD206 percent surface expression on differentiated MO-MФ isolated from WT or miR-15a/16-1/- B6 mouse spleens treated ex vivo with M-CSF (100 U/mL) and IL-4 $(20 \mathrm{ng} / \mathrm{mL})$ for 48 hours (A) or 6 days (B). Gating strategy was set using an IgC anti-Alexa Fluor 488 antibody

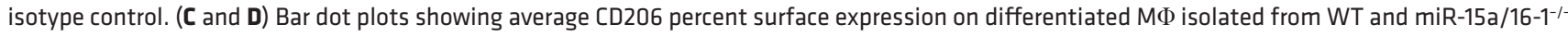
mice treated for 48 hours ( $n=4$ mice/group) (C) or 6 days ( $n=3$ mice/group) (D). (E and F) Parallel to $\mathbf{A}$ and $\mathbf{B}$, except that the surface marker analyzed was Dectin-1 and the gating strategy was set using an IgC anti-APC antibody isotype control. (G and $\mathbf{H}$ ) Parallel to $\mathbf{C}$ and $\mathbf{D}$, except that percents of Dectin-1 expression were recorded. Values represent the mean $\pm \mathrm{SD} ; P$ values were calculated using 2-tailed unpaired $t$ test. $P$ values are reported in the figures. 
A Primary MM-BM-CD14+ cells

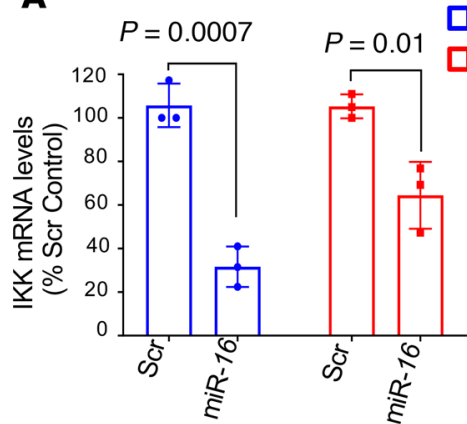

D

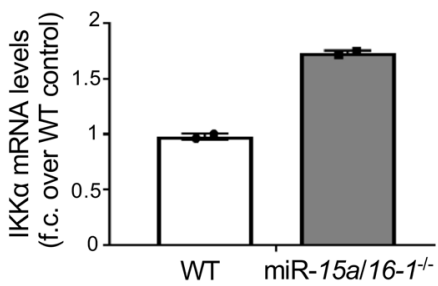

B

$K \alpha$

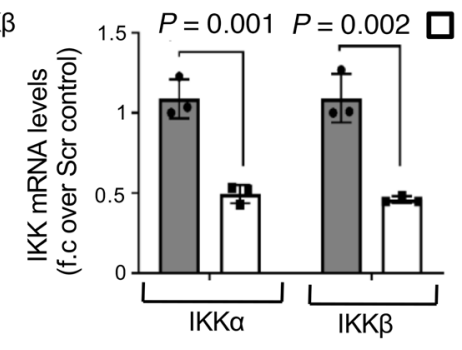

E

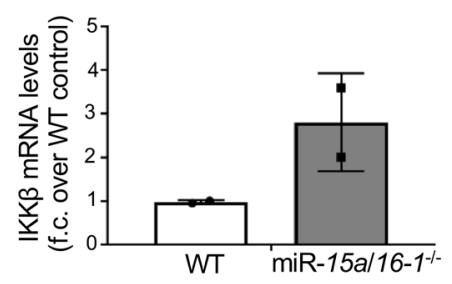

G

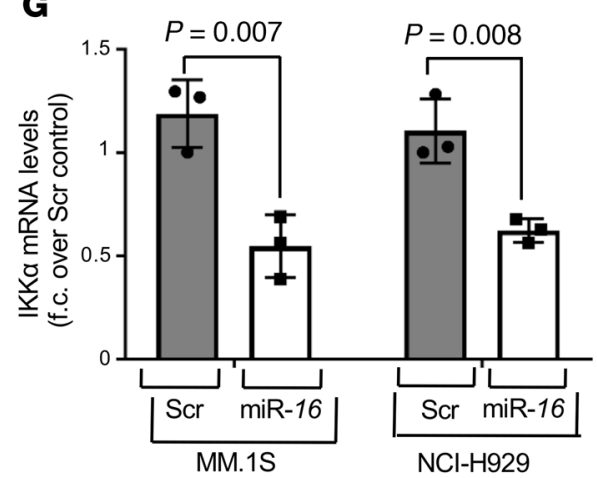

H MM.1S

Scr miR-16

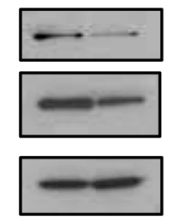

NCl-H929

Scr miR-16

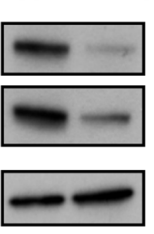

$\mathbf{F}$
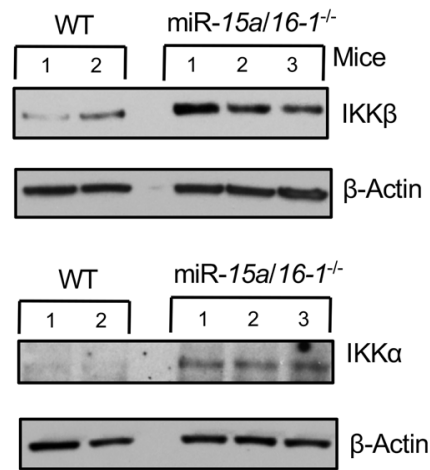

U266

Scr miR-16

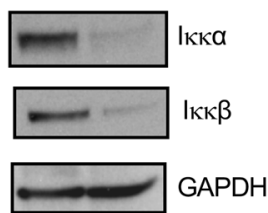

\section{I}

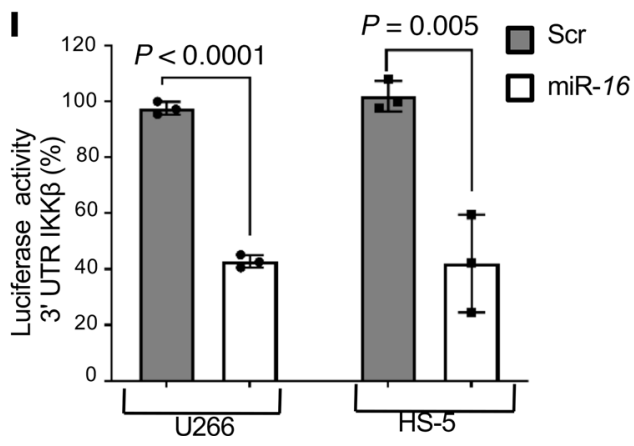

Figure 6. MiR-16 directly regulates the expression of IKK $\alpha / \beta$ complex (A and $B$ ) Real-time PCR analysis revealing mRNA expression of IKK $\alpha$ and $-\beta$ complex (A) in MФ isolated from MM patients $(n=3)$, and $U-937$ cell line (B), each transfected with ds miR-16 or Scr control for 48 hours. (C) Western blot analysis showing protein downregulation of IKK $\alpha$ and $-\beta$ by miR-16 in U-937 (left panel) and HS- 5 cells (right panel) as compared with Scr control after 48 hours of treatment; CAPDH was used as loading control. (D and $\mathbf{E}$ ) Real-time PCR showing mRNA expression

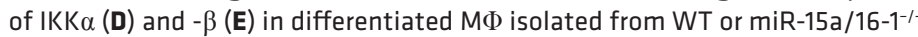
mouse spleens ( $n=4$ /group; the RNA from each 2 mice/group were pooled for quality purposes and a triplicate PCR reading was done for each pool). In $\mathbf{D}$ and E, error bars represent standard deviation. (F) Western blot analysis showing protein levels of IKK $\beta$ and IKK $\alpha$ in monocytes/MФ CD11b fractions isolated from WT or miR-15a/16-1 $1^{-1-}$ mice spleens ( $n=2$ mice, WT; $n=3$ mice, miR-15a/16-1/-). $\beta$-Actin is used as loading control. (C) Real-time PCR showing mRNA downregulation of IKK $\alpha$ expression in MM cell lines (MM.1S and NCI-H929) transfected with ds-miR-16 as compared with Scr control for 48 hours. (H) Western blot analysis showing protein downregulation of IKK $\alpha$ and $-\beta$ by miR-16 in 3 MM cell lines (U266, MM.1S, and NCI-H929); GAPDH was used as loading control. (I) Luciferase reporter assay revealed direct downregulation of IKK $\beta 3^{\prime} U$ UTR transcriptional activity by miR-16. pGL4.11 luciferase vector containing the $3^{\prime}$ UTR of IKK $\beta$ was transfected in U266 or HS-5 for 18 hours, followed by a second transfection with ds-miR-16 or Scr control for an additional 12 hours. Transfection efficiency was controlled by cotransfection with TK promoter-Renilla vector. Data are presented as percent of Scr control. When not otherwise specified in the legend, the reported $P$ values were calculated using 2-tailed unpaired $t$ test. Each of the reported experiments was performed in triplicate. For $\mathbf{C}$ and $\mathbf{H}$, the data presented are representative of 2 independent experiments. 
A Scr miR-16

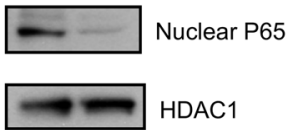

D

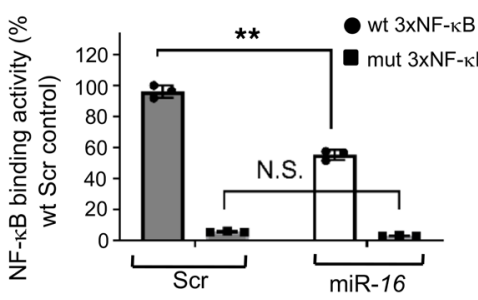

E
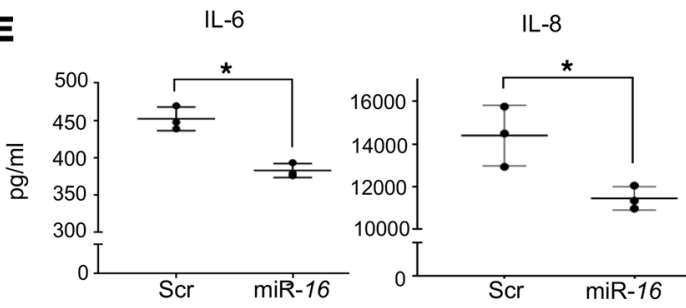

$\mathbf{B}$
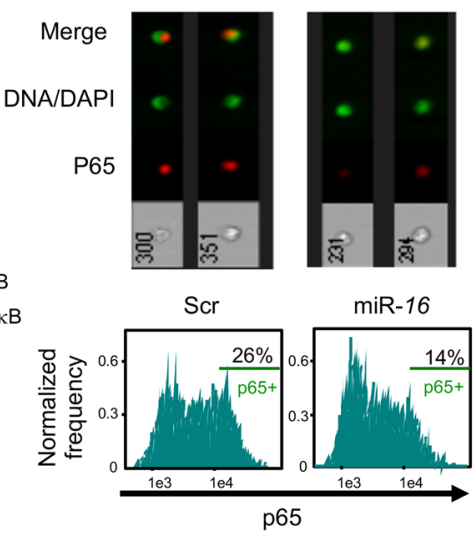

IL-8 TNF- $\alpha$
C

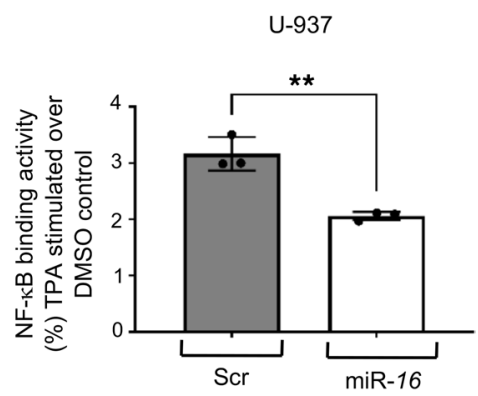

INF- $\gamma$
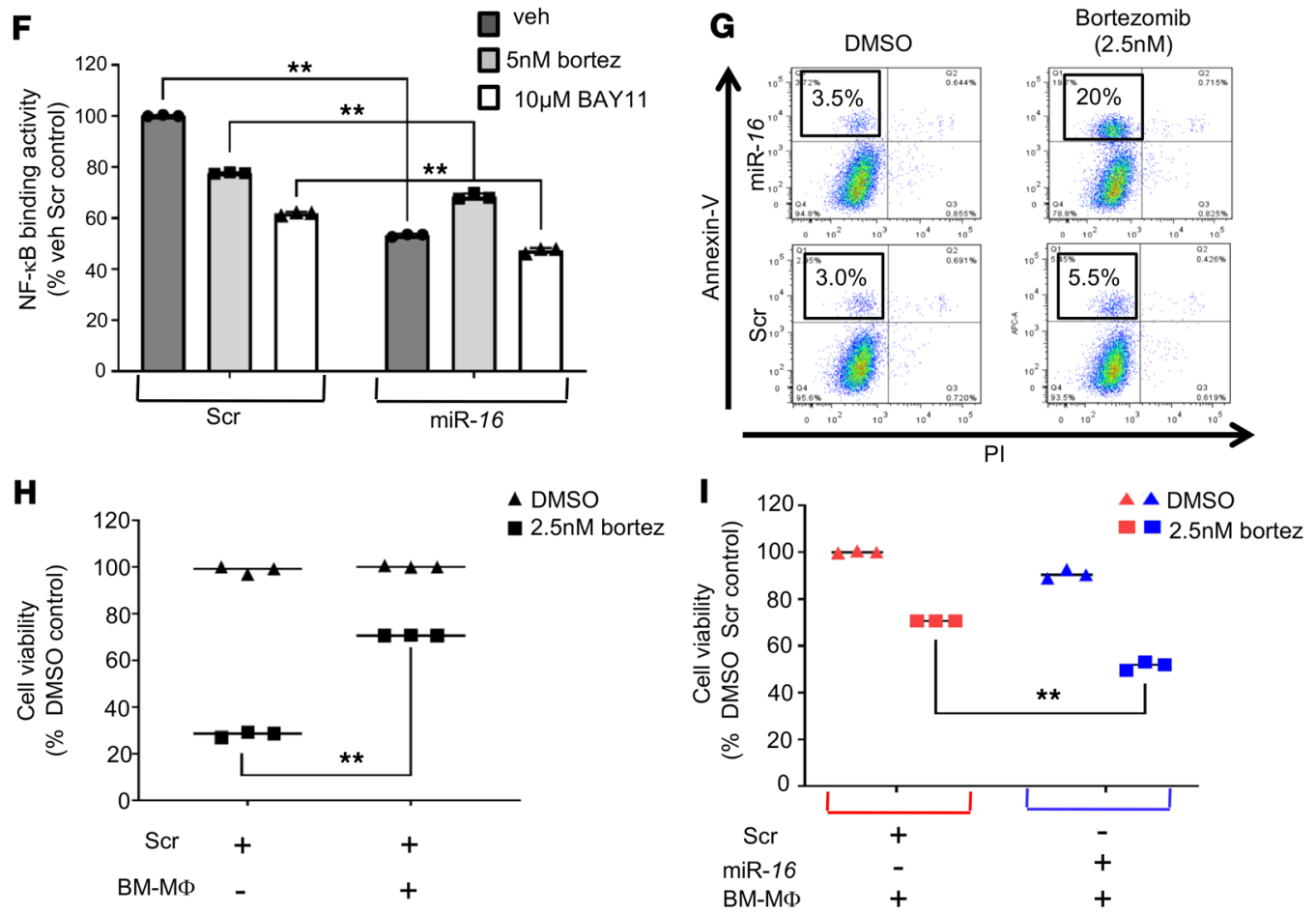

Figure 7. 36. MiR-16 increases MM cell sensitivity to bortezomib by impairing NF-кB signaling. (A) Western blot analysis showing decrease in P65 nuclear protein in NCl-H929 upon transfection with ds-miR-16 or Scr Control for 48 hours. Histone Deacetylase 1 (HDAC1) was used as nuclear loading control. (B) Single cell flow cytometric analysis showing decrease in nuclear p65 protein expression in MM cells upon transfection with ds-miR-16 as compared with Scr control (upper panel). Percent of normalized P65 frequency in $1 \times 10^{4}$ cells transfected with ds-miR-16 or Scr control are shown (lower panel). (C) Luciferase reporter assay showing decrease in NF- $\mathrm{BB}$ transcriptional activity in U-937 cells by miR-16 as compared with Scr control. (D) Parallel to C, except that luciferase activity was assessed in HS-5 cells, no stimulation was induced, and an additional transfection with pGL3 vector containing a trimer of mutated NF- $\kappa B$ binding sites sequence (mut $3 \times N F-\kappa B$ ) was performed. Data are presented as percent of WT $3 \times N F-\kappa B$ Scr control. (E) Cytokine array showing concentrations of cytokines/chemokines IL-6, IL-8, TNF- $\alpha$, and INF- $\gamma$ released by BM-MФ isolated from MM patient upon treatment with ds-miR-16 or Scr sequences encapsulated in liposomes for 48 hours. (F) Luciferase reporter assay showing decrease in NF- $\kappa B$ transcriptional activity in U266 upon transfection with miR-16 as compared with Scr control. Cells were transfected with pGL3 luciferase vector containing a trimer of consensus WT NF- $\kappa B$ binding sites for 6 hours and were then treated with $5 \mathrm{nM}$ bortezomib (bortez), $10 \mu \mathrm{M}$ BAY11-7082, or vehicle control (veh) for 12 hours. Data are presented as percent of Scr veh control. (G) Representative flowsight showing apoptosis analysis by Annexin-V/PI double staining. Annexin-V/PI staining was performed on MM.1S 
cells upon transfection with miR-16 or Scr sequences and treated with bortez $2.5 \mathrm{nM}$ or veh DMSO control for 48 hours. The percentages of Annexin-V-positive cells are shown in the upper left quadrants. (H) Luciferase assay showing percent of viable MM.1S GFP/Luc cells in suspension upon treatment with bortez or DMSO veh control, cocultured with BM-MФ isolated from a patient for 48 hours. (I) Luciferase assay showing percentage of viable MM.1S GFP/ Luc $^{+}$cells in suspension upon treatment with bortez or DMSO veh control cocultured with BM-MФ isolated from a MM patient and treated with ds-miR-16 or Scr sequences for 48 hours. Values represent the mean \pm SD for each experiment performed in triplicate. When not otherwise specified in the legend, the reported $P$ values were calculated using 2-tailed unpaired $t$ test.

6I). In support of IKK $\beta$-specific miR-16 targeting, a reporter vector carrying an IKK $\beta$ 3'UTR miR-16 mutated seed sequence was not significantly affected by miR-16 ectopic expression (Supplemental Figure 4, C and D), supporting the idea that miR-16 can directly regulate IKK $\beta$. Taken together, our data indicate that the mechanism of action through which miR-16 produces its effects is the direct targeting of the IKK $\alpha / \beta$ complex.

MiR-16 increases MM cell sensitivity to bortezomib by impairing the NF- $\kappa B$ signaling pathway. Because IKK $\alpha$-IK-

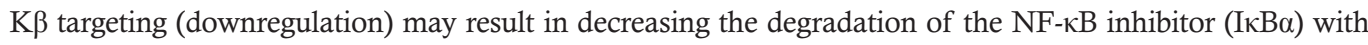
subsequent inhibition of p65/p50 transcriptional activation (40), we investigated whether differential miR-16 expression may regulate this process. Western blot data and single cell flow cytometry show that miR-16 inhibits p65 translocation to the nucleus (Figure 7, A and B). To determine whether miR-16 inhibits NF- $\mathrm{kB}$ transcriptional activity, we performed transient assays using a reporter plasmid containing 3 tandem WT P65/P50-binding sites upstream of a luciferase gene. U-937 cells were transfected with the reporter, and $\mathrm{NF}-\kappa \mathrm{B}$ activation was induced by treatment with tissue plasminogen (TPA). We found that, whereas scramble induced an almost 3-fold change, miR-16 induced a 2-fold change in NF- $\kappa B$ binding activity (Figure 7C). Additionally, the same effect of miR-16 was seen when ectopically expressed in HS-5, which is known to have a constitutively active NF- $\mathrm{kB}$ pathway (around 2-fold decrease in activity; ref. 41) (Figure 7D). However, miR-16 did not block the activity of a reporter containing 3 tandem mutated NF- $\mathrm{kB}$ sites, indicating that it specifically inhibits the canonical NF- $\mathrm{kB}$ pathway and that the inhibition does not block general transcriptional responses (Figure 7D). These results demonstrate that miR-16 not only acts to inhibit the NF- $\mathrm{kB}$ signaling

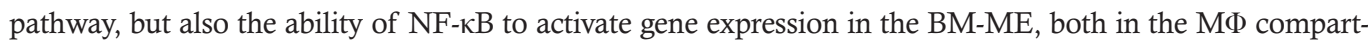
ment and stromal cells. As a result, miR-16 significantly downregulated the release of NF- $\mathrm{BB}-\mathrm{dependent}$ M2-like tumor-promoting cytokines/chemokines, IL-6, IL-8, and TNF- $\alpha$, from BM-MФ and upregulated the levels of IFN- $\gamma$ (Figure 7E). IFN- $\gamma$ has been previously shown to prevent the generation of TAM (42).

Because NF- $\mathrm{BB}$ is highly activated in MM cells (12), we tested the combinatorial effect of miR-16 with a PI (bortezomib) or the NF- $\mathrm{BB}$ inhibitor BAY11-7082 in MM cells. We found that ectopic expression of miR16 had a significant additive effect in inhibiting NF- $\mathrm{B}$ transcriptional activity when MM cells were treated with bortezomib or BAY11-7082 (Figure 7F). At the molecular level, combination treatment of miR-16 and bortezomib increased cytoplasmic levels of $\mathrm{I} \kappa \mathrm{B} \alpha$, the inhibitor of NF- $\mathrm{B}$, in $3 \mathrm{MM}$ cell lines tested (MM.1S, NCI-H929, and RPMI-8226) after 8 hours of treatment (Supplemental Figure 5A). To evaluate the clinical relevance of our findings, we examined whether miR-16 can sensitize MM-PCs and/or resident MФ of the BM-ME to bortezomib. We first examined the combinatorial effect of miR-16 with low doses of bortezomib on an MM cell line, MM.1S, in vitro. We found that cotreatment of miR-16 and bortezomib induced an additive decrease in cell viability in a dose-dependent manner (Supplemental Figure 5B). Ectopic expression of miR-16 alone did not induce cell death in MM.1S, whereas in combination with low doses of bortezomib, miR-16 sensitized MM cells to early apoptosis, as the combination treatment increased annexin V-positive cells from $5.5 \%$ to $20 \%$, in contrast to results from Scr control (Figure $7 \mathrm{G}$ ). However, in the context of the BM-ME, it has been previously shown that M2 MФ support MM cell survival and render them resistant to up to $5 \mathrm{nM}$ bortezomib in culture $(28,43)$. Treatment of MM cells alone with low doses of bortezomib induced a decrease in cell viability, whereas in the presence of resident $\mathrm{M} \Phi$, the sensitivity was significantly abrogated (Figure $7 \mathrm{H}$ ). For that reason, we investigated whether the combination treatment of miR-16 and bortezomib would sensitize MM.1S to bortezomib in the presence of BM-resident MФ isolated from a MM patient. To specifically measure the viability of MM cells in coculture systems, we used MM cells that constitutively express the luciferase gene (MM.1S GFP/Luc ${ }^{+}$), and we measured their viability by luciferase activity (Figure 7, $\mathrm{H}$ and

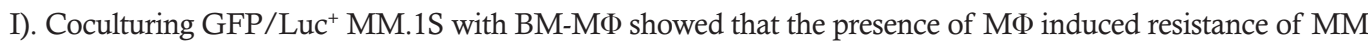
cells to cell death (Figure $7 \mathrm{H}$ ). However, the attenuation of bortezomib-based anti-MM activity by MФ was significantly reversed by ectopic overexpression of miR-16 in both MФ and MM cells (Figure 7I). Taken together, our results show that miR-16 inhibits NF- $\mathrm{kB}$ transcriptional activity both in MM cells and the BM-ME and potentiates the activity of a PI in a cross-talk manner by affecting both MM cells and BM-resident MФ. 


\section{Discussion}

The BM-ME plays an important role in MM progression and response to therapy (9). MM cells interact with the BM-ME components in a paracrine manner, either by direct cell-to-cell interaction or indirect communication through release of soluble factors, as well as EVs (44). Here, we show that EVs released by MM cells induce monocyte differentiation toward an M2 phenotype. Our previous results showed that EVs released by MM cells are enriched in the MHC-1 antigen presenting complex and its binding protein, $\beta 2-\mathrm{M}(26,45,46)$, and recent published data reported that this complex plays a pivotal role in inducing cancer cell protection from $\mathrm{M} \Phi$ phagocytosis (47). Here, we found that EVs isolated from MM patients' BM-ac induce monocyte polarization toward an M2 phenotype. It has been reported that EVs derived from MM cells carrying miR-21 and miR-146a induce mesenchymal stromal cell proliferation and transformation, resulting in MM cell growth promotion and migration (48). We and others have shown that MM patients with high levels of circulating miR-16 have longer survival and that circulating miR-16 levels were reduced in the serum of MM patients carrying Del13 in their cancer cells as compared with levels associated with non-Del13 or normal PCs $(26,49)$, supporting that changes in chromosome 13 status may not only affect the intracellular landscape of cancer cells, but also their tumor ME. Although a negative correlation between $\mathrm{miR}-15 \mathrm{a} / 16-1$ levels and the expression of the MM supportive cytokine IL-6 has been reported $(24,50)$, the molecular mechanisms behind this effect were not clearly investigated (50). We demonstrate that extracellular miR-16 can impair the ability of MM EVs to differentiate monocytes to $\mathrm{M} 2-\mathrm{M} \Phi$ and that ectopic expression of miR-16 directly downmodulates NF- $\mathrm{kB}$ signaling. Specifically, we found that miR-16 is not only able to target IKK $\alpha$ as previously reported (51), but it is also the key catalytic kinase of the NF- $\mathrm{kB}$ canonical pathway, IKK $\beta$, in MM cells and also in $\mathrm{M} \Phi$ and BM stromal cells. We demonstrate that ectopic expression of miR-16 impairs monocyte differentiation to M2-MФ and the subsequent release of M2-associated cytokines/chemokines such as IL-6, IL-10, IL-8, and TNF- $\alpha$ (Figure 8). We also show that Del13, in addition to affecting the intracellular genetic landscape of MM cells as previously reported $(16,17)$, also impacts the surrounding BM-ME, creating a clonal advantage of cancer cells carrying this deletion versus the WT form. The importance of miR-16 in impairing M2-MФ differentiation through IKK $\beta$ targeting is also supported by previously published data showing that the NF- $\mathrm{kB}$ canonical pathway alone is essential to maintain an $\mathrm{M} 2$ phenotype, as only the MФ isolated from IKK $\beta-\mathrm{KO}$ mice could be reeducated to have antitumor activity, in contrast to those isolated from WT mice (52). By using a miR-15a/16-1-KO mouse model (21), we also show that miR15a/16-1-KO MФ had a significantly stronger M2 phenotype, as indicated by the M2 surface markers CD206 and Dectin-1, compared with that in WT mice. These findings are also consistent with recently published data in which a miR-15-16-double KO model exhibits myeloid disorders (53). Finally, we showed the clinical benefit of miR-16 in sensitizing MM cells to bortezomib treatment. Our findings align with a clinical study that showed exosomal miR-16 downregulation in patients with bortezomib resistance (54). We found that miR-16 not only directly sensitized MM cells to bortezomib in an autologous manner, but also reverted their PI resistance when myeloma cells were cocultured in the presence of TAMs isolated from the BM aspirates of MM patients, further supporting that TAMs provide a survival benefit to MM cells (43). In summary, we show that EVs released by MM cells can affect the tumor ME by modulating the polarization of circulating monocytes to M2-M which fully resembled the BM-MФ characteristics found in patients. Our data also show that miR-16 can downmodulate the NF- $\kappa \beta$ pathway in PCs and the microenvironment, in which activation is critical for maintaining MM-PC survival during early-stage disease (14). Our data provide the scientific rationale for the manner in which miR-16 downmodulation provides a survival advantage to specific PC clones during MM progression.

\section{Methods}

Supplemental methods are available online with this article.

Primary samples. Primary samples (total BM aspirates and peripheral blood samples) from MM patients and healthy donors were obtained from The Ohio State University and City of Hope Leukemia Tissue Banks. Specifically, the cellular fraction of total BM aspirates was isolated using Ficoll-Paque Plus (catalog 17144003, GE Healthcare) following the manufacturer's instructions.

Cell lines. MM cell lines (MM.1S, NCI-H929, U266, and RPMI-8226), the U-937 MФ cell line, the BM stromal cell line HS-5, and the HeLa cell line were purchased from ATCC. The GFP $/ \mathrm{Luc}^{+} \mathrm{MM} .1 \mathrm{~S}$ stable line was a gift from Irene Ghobrial (Dana-Farber Cancer Institute, Boston, Massachusetts, USA). OPM2, LP-1, L363, OCIMY-I, OCIMY-5, and MMM.1 MM cell lines were provided by Jonathan J. Keats (https://www.keatslab.org/). MM and U-937 cell lines were cultured in RPMI-1640 medium supplemented with $10 \%$ FBS (catalog 019K8420, MilliporeSigma), $100 \mathrm{IU} / \mathrm{mL}$ penicillin, and $100 \mu \mathrm{g} / \mathrm{mL}$ streptomy- 


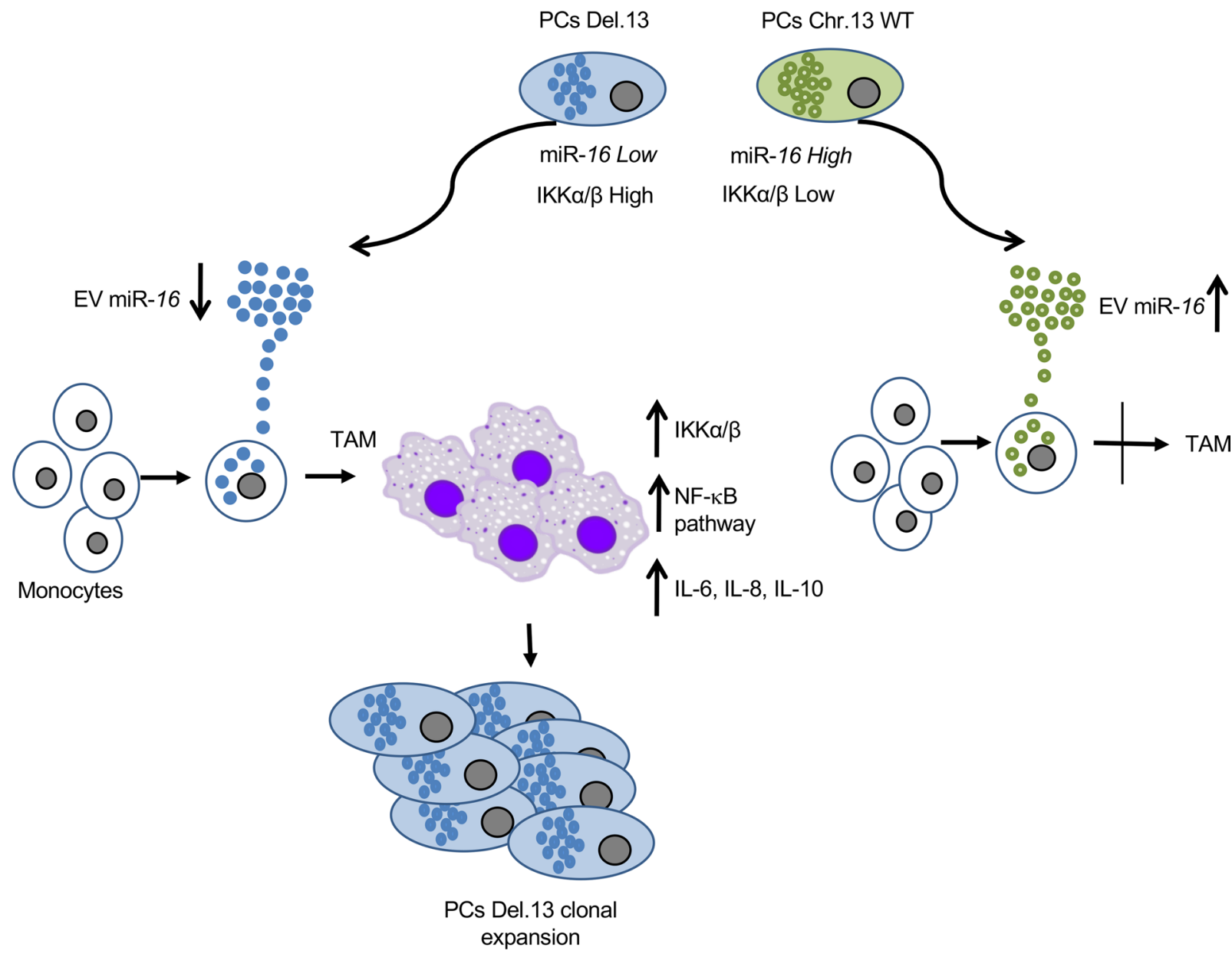

Figure 8. Illustrative diagram showing plasma cells carrying Del13 (PCs Del13) secrete extracellular vesicles (EV) containing cargoes that induce monocyte differentiation toward M2 tumor-supporting M (TAM). Mechanistically, a lack of significant amounts of miR-16 in EV prevents targeting the IKK $\alpha / \beta$ complex, resulting in an increase in the NF- $\kappa B$ canonical pathway. Upregulation of the NF- $\kappa B$ pathway leads to enhanced secretion of M2 tumor effector chemokines/ cytokines, including IL-6, IL-8, IL-10, and TNF- $\alpha$, resulting in an increase in TAM levels and in PC clonal expansion. However, normal PCs or PCs carrying WT chromosome 13 (PCs Chr.13 WT) have higher levels of miR-16 in their EV that target the IKK $\alpha / \beta$ complex, resulting in a decrease in the NF- $\kappa B$ canonical pathway and impairment of monocyte differentiation toward the M2-M subset of the microenvironment and, thus, PC clonal expansion.

cin (catalog 15140-122, Gibco). The HS-5 cell line was cultured in DMEM supplemented with 10\% FBS, $100 \mathrm{IU} / \mathrm{mL}$ penicillin, and $100 \mu \mathrm{g} / \mathrm{mL}$ streptomycin. HeLa cells were cultured in EMEM supplemented with $10 \% \mathrm{FBS}, 100 \mathrm{IU} / \mathrm{mL}$ penicillin, and $100 \mu \mathrm{g} / \mathrm{mL}$ streptomycin.

Cell transfection. HS-5 or HeLa cell lines were transfected with Lipofectamine 3000 Transfection Reagent (catalog L3000-008, Invitrogen) following the manufacturer's instructions. MM cell lines were transfected by electroporation using the Nucleofector4D system (Lonza). Specific nucleofection solutions and programs were optimized for each cell line. Briefly, on day 1 , two batches of $6 \times 10^{6}$ cells were each resuspended in 100 $\mu \mathrm{L}$ of the nucleofector SF solution containing 50 pmol microRNA hsa-miR-16-5p mimic (Thermo Fisher Scientific, catalog 4464066) or scramble control (Thermo Fisher Scientific, catalog 4464058) and transferred to a cuvette. Each electroporated batch of cells were then kept in culture in $10 \mathrm{~mL}$ complete growth media for the next day. On day 2, the 2 batches of cells were combined and transfected again following the same procedure. On day 3, cells were harvested for further experiments. Program DS-137 was used for MM.1S cells, program DN-100 for U266, program DS-150 for RPMI-8226, and program CM-138 for NCI-H929. All MM cell lines were transfected 2 times unless indicated otherwise in the figure legends. The U-937 cell line was transfected by electroporation using the Nucleofector4D system (Lonza) according to the manufacturer's instructions. Primary cells were transfected using DOTAP Liposomal Transfection Reagent (catalog 144189-73-1, Sigma-Aldrich) according to the manufacturer's instructions. The hsa-miR-223 mimic microRNA used was hsa-miR-223-5p mimic (Thermo Fisher Scientific, catalog 4464066). 
$m R N A$ and miRNA expression. Quantitative PCR (qPCR) was performed with the TaqMan method (Applied Biosystems), according to the manufacturer's instructions, and analyzed with the 7900HT Sequence Detection System (Applied Biosystems). The appropriate TaqMan probes for mRNA and miRNA quantification were purchased from Applied Biosystems, and all reactions were performed in triplicate. The following probes were used: hsa-miR-16-5p (477860_mir), hsa-miR-142-3p (000464_mir), hsa-miR-9 (000583_mir), hsa-CD163 (Hs00174705_m1), hsa-IRF4 (Hs00180031_m1), hsa-CD80 (Hs01045161_m1), hsa-IKK $\alpha$ (Hs00989497_m1), hsa-IKK $\beta$ (Hs01559460_m1), mm-IL-10 (Mm01288386_m1), mm-IL-12 (Mm00434169_m1), mm-IKK $\alpha$ (Mm00432529_m1), and mm-IKK $\beta$ (Mm01222247_m1). GAPDH (Hs99999905_m1) was used as a reference for human mRNA data normalization, while small endogenous nucleolar RNA RNU44 (001094) was used for miRNA normalization. Simultaneous quantification of ornithine decarboxylase antizyme 1 (OAZ1) (Mm00814770_g1) was used as a reference for mouse mRNA data normalization. The relative expression levels were calculated by the comparative Ct method (User Bulletin \#2; Applied Biosystems). EV miRNA isolation for qPCR analysis was done using the exoEasy Maxi Kit (catalog 76064, QIAGEN) according to the manufacturer's instructions.

Monocyte differentiation. $\mathrm{CD}^{+}{ }^{+}$cell isolation from primary samples, either BM or peripheral blood, was done using CD14 microbeads (catalog 130-050-201, Miltenyi Biotec) according to the manufacturer's instructions. Isolated cells were differentiated for 7 days with granulocyte MФ CSF (GM-CSF) (20 ng/ $\mathrm{mL}$ ) or MФ CSF (M-CSF) (20 ng/mL) added every 2 days to generate in vitro M1 or M2-M Differentiation of $\mathrm{CD}_{14}{ }^{+}$cells using EV isolated from MM cells was done at a ratio of 1:8 in culture for the time indicated in the figure legends. Differentiation of $\mathrm{CD} 14^{+}$cells with the $\mathrm{BM}-\mathrm{ac}$ or $\mathrm{BM}$ conditioned media was done at the ratio of $1 \times 10^{6} \mathrm{CD} 14^{+}$cells $/ 1 \mathrm{~mL}$ of $\mathrm{BM}$-ac in culture for the times indicated in the figure legends. Formally, we categorized the BM-ac as the human BM plasma, which mainly consists of dissolved proteins (e.g., chemokines, cytokines, growth factors), hormones, carbon dioxide, and EVs. It makes up approximately $55 \%$ of the whole $\mathrm{BM}$ suspension. This whole BM plasma is isolated from a single donor. Briefly, the plasma is separated out from the whole BM by centrifugation $(2,000 \mathrm{~g}$ for 20 minutes at room temperature) in anticoagulant (EDTA) coated vials. The upper, lighter yellowish layer, which is the plasma, is collected and immediately frozen at $-80^{\circ} \mathrm{C}$ in order to preserve its contents. We instead used the term "BM conditioned media" when the entire BM cellular populations were cultured in exosome-free media, and the supernatant was collected after 48 hours of culture.

Animal models. Mice were housed under a 12-hour light-dark cycle with food and water ad libitum. miR-15a/16-1-KO mice (C57BL/6 background) were a gift from R. Dalla-Favera (Columbia University, New York, New York, USA) (21). Monocytes/MФ were isolated using the CD11b microbeads isolation kit (catalog 130-049-601, Miltenyi Biotec).

Isolation and ex vivo treatment of mouse spleen $M \Phi$. Spleenocytes were isolated from healthy C57BL/6 WT or miR-15a/16-1-KO mice (21), and M0 MФ were isolated using anti-mouse F4/80 MicroBeads isolation kit (catalog 130-110-443, Miltenyi Biotec) according to the manufacturer's instructions. F4/80 $0^{+}$cells were cultured ex vivo at a density of $1 \times 10^{6}$ cells $/ \mathrm{mL}$ in DMEM/F12-10 supplemented with $10 \%$ FBS and 100 $\mathrm{U} / \mathrm{mL}$ of recombinant murine M-CSF (catalog 315-02, PeproTech) and incubated at $37^{\circ} \mathrm{C}$ with $5 \% \mathrm{CO}_{2}$. To induce MФ differentiation to the M2 phenotype, at 8 hours after isolation, $20 \mathrm{ng} / \mathrm{mL}$ of murine IL-4 was added and replenished every 2 days. Cells were harvested at both 48 hours and 6 days of culture for detection of CD206 and Dectin-1 surface markers by flow cytometry.

EV isolation. The method used for isolation of cell line-derived EVs was previously described by Théry et al. (55). In short, serum-starved cells and media were centrifuged at $300 \mathrm{~g}$ for 10 minutes at $4^{\circ} \mathrm{C}$. Supernatant was collected and centrifuged again at 2,000 $\mathrm{g}$ for 20 minutes at $4^{\circ} \mathrm{C}$. The cell pellets were frozen and stored at $-80^{\circ} \mathrm{C}$ for later use. Supernatant was harvested and vacuum ultracentrifuged at $10,000 \mathrm{~g}$ for 30 minutes at $4^{\circ} \mathrm{C}$ to remove residual cell debris. Supernatant was collected and ultracentrifuged at 100,000 $g$ for 70 minutes at $4^{\circ} \mathrm{C}$ under vacuum. The resulting supernatant was discarded. Pellets from multiple tubes were resuspended in $1 \mathrm{~mL}$ of PBS, pooled into a single tube, and ultracentrifuged at $100,000 \mathrm{~g}$ as described previously (45). Supernatant was eliminated, and pellets of vesicles were resuspended in the desired amount of PBS and used for monocytes treatment.

miRNA microarray analysis. Intracellular or EV miRNA levels were measured as previously described (26). Briefly, $1 \mu \mathrm{g}$ RNA isolated from cells or EVs released by cells was analyzed by NanoString assay performed as described by the manufacturer (NanoString Technologies Inc.). NanoString raw data, which were proportional to copy number, were log-transformed and normalized by the quan- 
tile method. $P$ values were used to rank miRNAs of interest, and correction for multiple comparisons was done using the Benjamini-Hochberg method.

DNA constructs. A trimer of WT human NF- $\kappa \mathrm{B}$ binding site $(\mathrm{WT} 3 \times \mathrm{NF}-\kappa \mathrm{B})$ and a trimer of human mutated NF- $\mathrm{B}$ binding site in pGL3 luciferase vectors were gifts from Denis Guttridge (The Ohio State University). For generation of a human 3'UTR IKK $\beta$ luciferase reporter construct containing a miR-16-5p WT binding site, $249 \mathrm{bp}$ were amplified and cloned into the XbaI site of pGL4.11 luciferase construct (Promega) using the following primers: (forward) 5'-ATCTAGATCCCTGTCCTCTCTCACTTTAC-3' and (reverse) 5'-ATCTGAGGCTCTCCCATCCTGGTTACTAT-3'

A mutated 3'UTR IKK $\beta$ construct was generated by mutating the miR-16-5p binding site using QuikChange Site-Directed Mutagenesis Kit (Agilent Technologies) according to the manufacturer's instructions and the following primers: (forward) 5'-CTTCCTCTTTTTATTTCACTGGATCCAAAATTGTGTTTTTACCTAC-3' and (reverse) 5'-GTAGGTAAAAACACAATTTTGGATCCAGTGAAATAAAAAGAGGAAG-3' .

Mutations involve introduction of the Bam $\mathrm{HI}$ sites underlined above.

Statistics. All quantitative data were presented when indicated as the mean $\pm \mathrm{SD}$, and at least 3 experiments were performed. GraphPad Prism 8 was used for data analysis. Experimental data with a normal distribution between 2 comparison groups were analyzed by unpaired 2-tailed $t$ test. One-way ANOVA was used to analyze experiments with multiple comparisons ( $>2$ groups). The post-test value is reported for each analysis.

Study approval. All animal studies were approved by the City of Hope IACUC. Our studies involving human subjects (NCT01408225) were approved by the IRB of The Ohio State University or City of Hope and conformed to the tenets of the Declaration of Helsinki. Participants provided informed written consent.

\section{Author contributions}

JK performed experiments, analyzed the data, and wrote the manuscript. JG, MM, DV, ET, and EGG performed experiments. CT and AS analyzed the single cell flow cytometry data. AR performed experiments and revised the manuscript. PLB, MC, SR, GM, CCH, and AK scientifically contributed to the experimental design and revised the manuscript. AP and JJK performed bioinformatics analyses. AD helped prepare and design the figures. JFS scientifically corrected the manuscript. EC performed experiments and helped supervise the project. FP designed and directed the project and wrote the manuscript.

\section{Acknowledgments}

Research was, in part, supported by the NIH under grant numbers NIH-2-R01-CA201382 and NIH- 2-R01CA194742 (CCH, FP) and by the MMORE for Multiple Myeloma Research Fund. We thank Robert Weingart and Ramasamy Santhanam for their support in accomplishing the experimental work. We also thank R. Dalla-Favera for providing us with the miR-15a/16-1 $1^{-/-}$mouse model and Irene Ghobrial for providing us with the MM.1S GFP/Luc ${ }^{+}$cell line. We thank The Ohio State University Leukemia Tissue Bank (NCT01408225) for primary sample collection under the supervision of Craig C. Hofmeister. We thank Wei Chen and Xiwei Wu at the City of Hope integrative genomics core for bioinformatics support. We would also like to thank Lucy Ghoda and Tinisha McDonald for primary sample collection from City of Hope Leukemia Tissue Bank. We recognize Elizabeth Hartman, Evelyn Flores, and Debbie Flood for administrative support. Research reported in this publication included work performed at the City of Hope Liquid Tissue Bank, Analytical Cytometry, and Integrative Genomics and Bioinformatics shared resource cores supported by the NIH under award number P30CA033572. The content is solely the responsibility of the authors and does not necessarily represent the official views of the NIH.

Address correspondence to: Flavia Pichiorri, 1500 East Duarte Road, Duarte, California 91010, USA. Phone: 626.218.4908; Email: fpichiorri@coh.org. Or to: Enrico Caserta, 1500 East Duarte Road, Duarte, California 91010, USA. Phone: 626.218.8519; Email: ecaserta@coh.org.

1. Jemal A, Siegel R, Xu J, Ward E. Cancer statistics, 2010. CA Cancer J Clin. 2010;60(5):277-300.

2. Fonseca R, San Miguel J. Prognostic factors and staging in multiple myeloma. Hematol Oncol Clin North Am. 2007;21(6):1115-1140.

3. Fonseca R, et al. International Myeloma Working Group molecular classification of multiple myeloma: spotlight review. Leukemia. 2009;23(12):2210-2221.

4. Weiss BM, Abadie J, Verma P, Howard RS, Kuehl WM. A monoclonal gammopathy precedes multiple myeloma in most patients. Blood. 2009;113(22):5418-5422.

5. Weiss BM, et al. Patterns of monoclonal immunoglobulins and serum free light chains are significantly different in black com- 
pared to white monoclonal gammopathy of undetermined significance (MGUS) patients. Am J Hematol. 2011;86(6):475-478.

6. Weiss BM, Kuehl WM. Advances in understanding monoclonal gammopathy of undetermined significance as a precursor of multiple myeloma. Expert Rev Hematol. 2010;3(2):165-174.

7. Landgren O, Rajkumar SV. Development of early treatment strategies for high-risk myeloma precursor disease in the future. Semin Hematol. 2011;48(1):66-72.

8. Kim J, et al. Macrophages and mesenchymal stromal cells support survival and proliferation of multiple myeloma cells. $B r J$ Haematol. 2012;158(3):336-346.

9. Bianchi G, Richardson PG, Anderson KC. Promising therapies in multiple myeloma. Blood. 2015;126(3):300-310.

10. Di Marzo L, et al. Microenvironment drug resistance in multiple myeloma: emerging new players. Oncotarget. 2016;7(37):60698-60711.

11. Markovina S, et al. Bortezomib-resistant nuclear factor-kappaB activity in multiple myeloma cells. Mol Cancer Res. 2008;6(8):1356-1364.

12. Demchenko YN, Glebov OK, Zingone A, Keats JJ, Bergsagel PL, Kuehl WM. Classical and/or alternative NF-kappaB pathway activation in multiple myeloma. Blood. 2010;115(17):3541-3552.

13. Keats JJ, et al. Promiscuous mutations activate the noncanonical NF-kappaB pathway in multiple myeloma. Cancer Cell. 2007;12(2):131-144.

14. Demchenko YN, Kuehl WM. A critical role for the NFkB pathway in multiple myeloma. Oncotarget. 2010;1(1):59-68.

15. Calin GA, et al. Frequent deletions and down-regulation of micro- RNA genes miR15 and miR16 at 13 q14 in chronic lymphocytic leukemia. Proc Natl Acad Sci USA. 2002;99(24):15524-15529.

16. Zojer N, et al. Deletion of 13q14 remains an independent adverse prognostic variable in multiple myeloma despite its frequent detection by interphase fluorescence in situ hybridization. Blood. 2000;95(6):1925-1930.

17. Shaughnessy J, et al. High incidence of chromosome 13 deletion in multiple myeloma detected by multiprobe interphase FISH. Blood. 2000;96(4):1505-1511.

18. López-Corral L, et al. The progression from MGUS to smoldering myeloma and eventually to multiple myeloma involves a clonal expansion of genetically abnormal plasma cells. Clin Cancer Res. 2011;17(7):1692-1700.

19. Binder M, et al. Prognostic implications of abnormalities of chromosome 13 and the presence of multiple cytogenetic high-risk abnormalities in newly diagnosed multiple myeloma. Blood cancer journal. 2017;7(9):e600.

20. Avet-Loiseau H, et al. Monosomy 13 is associated with the transition of monoclonal gammopathy of undetermined significance to multiple myeloma. Intergroupe Francophone du Myélome. Blood. 1999;94(8):2583-2589.

21. Klein U, et al. The DLEU2/miR-15a/16-1 cluster controls B cell proliferation and its deletion leads to chronic lymphocytic leukemia. Cancer Cell. 2010;17(1):28-40.

22. Cimmino A, et al. miR-15 and miR-16 induce apoptosis by targeting BCL2. Proc Natl Acad Sci USA. 2005;102(39):13944-13949.

23. Liu J, et al. Loss of p53 and altered miR15-a/16-1 $\rightarrow$ MCL-1 pathway in CLL: insights from TCL1-Tg:p53(-/-) mouse model and primary human leukemia cells. Leukemia. 2014;28(1):118-128.

24. Roccaro AM, et al. BM mesenchymal stromal cell-derived exosomes facilitate multiple myeloma progression. J Clin Invest. 2013;123(4):1542-1555.

25. Lee JK, et al. Exosomes derived from mesenchymal stem cells suppress angiogenesis by down-regulating VEGF expression in breast cancer cells. PLoS ONE. 2013;8(12):e84256.

26. Rocci A, et al. Circulating miRNA markers show promise as new prognosticators for multiple myeloma. Leukemia. 2014;28(9):1922-1926.

27. Jia X, et al. MiR-16 regulates mouse peritoneal macrophage polarization and affects T-cell activation. J Cell Mol Med. 2016;20(10):1898-1907.

28. Zheng Y, et al. Macrophages are an abundant component of myeloma microenvironment and protect myeloma cells from chemotherapy drug-induced apoptosis. Blood. 2009;114(17):3625-3628.

29. Wang H, et al. High numbers of CD163+ tumor-associated macrophages correlate with poor prognosis in multiple myeloma patients receiving bortezomib-based regimens. J Cancer. 2019;10(14):3239-3245.

30. Baroni S, et al. Exosome-mediated delivery of miR-9 induces cancer-associated fibroblast-like properties in human breast fibroblasts. Cell Death Dis. 2016;7(7):e2312.

31. Tang J, et al. Exosomal miR-9-3p suppresses HBGF-5 expression and is a functional biomarker in hepatocellular carcinoma. Minerva Med. 2018;109(1):15-23.

32. Roccaro AM, et al. MicroRNAs 15a and 16 regulate tumor proliferation in multiple myeloma. Blood. 2009;113(26):6669-6680.

33. Manier S, Sacco A, Leleu X, Ghobrial IM, Roccaro AM. Bone marrow microenvironment in multiple myeloma progression. J Biomed Biotechnol. 2012;2012:157496.

34. Beider K, et al. Multiple myeloma cells recruit tumor-supportive macrophages through the CXCR4/CXCL12 axis and promote their polarization toward the M2 phenotype. Oncotarget. 2014;5(22):11283-11296.

35. Tran TH, Krishnan S, Amiji MM. MicroRNA-223 Induced Repolarization of Peritoneal Macrophages Using CD44 Targeting Hyaluronic Acid Nanoparticles for Anti-Inflammatory Effects. PLoS ONE. 2016;11(5):e0152024.

36. Schmid JA, Birbach A. IkappaB kinase beta (IKKbeta/IKK2/IKBKB)--a key molecule in signaling to the transcription factor NF-kappaB. Cytokine Growth Factor Rev. 2008;19(2):157-165.

37. Hagemann T, Biswas SK, Lawrence T, Sica A, Lewis CE. Regulation of macrophage function in tumors: the multifaceted role of NF-kappaB. Blood. 2009;113(14):3139-3146.

38. Sun SC. The noncanonical NF-кB pathway. Immunol Rev. 2012;246(1):125-140.

39. Agarwal V, Bell GW, Nam JW, Bartel DP. Predicting effective microRNA target sites in mammalian mRNAs. Elife. 2015;4:e05005.

40. Chen ZJ. Ubiquitination in signaling to and activation of IKK. Immunol Rev. 2012;246(1):95-106.

41. Manni S, et al. Bone marrow stromal cell-fueled multiple myeloma growth and osteoclastogenesis are sustained by protein kinase CK2. Leukemia. 2014;28(10):2094-2097.

42. Duluc D, et al. Interferon-gamma reverses the immunosuppressive and protumoral properties and prevents the generation of human tumor-associated macrophages. Int J Cancer. 2009;125(2):367-373. 
43. De Beule N, et al. Tumour-associated macrophage-mediated survival of myeloma cells through STAT3 activation. $J$ Pathol. 2017;241(4):534-546.

44. Kalluri R. The biology and function of exosomes in cancer. J Clin Invest. 2016;126(4):1208-1215.

45. Harshman SW, et al. Characterization of multiple myeloma vesicles by label-free relative quantitation. Proteomics. 2013;13(20):3013-3029.

46. Harshman SW, et al. Proteomic characterization of circulating extracellular vesicles identifies novel serum myeloma associated markers. J Proteomics. 2016;136:89-98.

47. Barkal AA, et al. Engagement of MHC class I by the inhibitory receptor LILRB1 suppresses macrophages and is a target of cancer immunotherapy. Nat Immunol. 2018;19(1):76-84

48. Cheng Q, et al. Multiple Myeloma-Derived Exosomes Regulate the Functions of Mesenchymal Stem Cells Partially via Modulating miR-21 and miR-146a. Stem Cells Int. 2017;2017:9012152.

49. Manier S, et al. Prognostic role of circulating exosomal miRNAs in multiple myeloma. Blood. 2017;129(17):2429-2436.

50. Hao M, et al. Suppressing miRNA-15a/-16 expression by interleukin- 6 enhances drug-resistance in myeloma cells. $J$ Hematol Oncol. 2011;4:37.

51. Li T, Morgan MJ, Choksi S, Zhang Y, Kim YS, Liu ZG. MicroRNAs modulate the noncanonical transcription factor NF-kappaB pathway by regulating expression of the kinase IKKalpha during macrophage differentiation. Nat Immunol. 2010;11(9):799-805

52. Hagemann T, et al. "Re-educating" tumor-associated macrophages by targeting NF-kappaB. J Exp Med. 2008;205(6):1261-1268.

53. Lovat F, et al. miR-15b/16-2 deletion promotes B-cell malignancies. Proc Natl Acad Sci USA. 2015;112(37):11636-11641.

54. Zhang L, et al. Potential role of exosome-associated microRNA panels and in vivo environment to predict drug resistance for patients with multiple myeloma. Oncotarget. 2016;7(21):30876-30891.

55. Théry C, Amigorena S, Raposo G, Clayton A. Isolation and characterization of exosomes from cell culture supernatants and biological fluids. Curr Protoc Cell Biol. 2006; Chapter 3:Unit 3.22. 